Prons

trobertivier

Journal of Nonlinear Mathematical Physics

\title{
Composition of Lie Group Elements from Basis Lie Algebra Elements
}

George W. Bluman, Omar Mrani-Zentar, Deshin Finlay

To cite this article: George W. Bluman, Omar Mrani-Zentar, Deshin Finlay (2018) Composition of Lie Group Elements from Basis Lie Algebra Elements, Journal of Nonlinear Mathematical Physics 25:4, 528-557, DOI:

https://doi.org/10.1080/14029251.2018.1503398

To link to this article: https://doi.org/10.1080/14029251.2018.1503398

Published online: 04 January 2021 


\title{
Composition of Lie Group Elements from Basis Lie Algebra Elements
}

\author{
George W. Bluman \\ Department of Mathematics, University of British Columbia, \\ Vancouver V6T 1Z2, Canada \\ bluman@math.ubc.ca \\ Omar Mrani-Zentar \\ Department of Mathematics, University of British Columbia, \\ Vancouver V6T 1Z2, Canada \\ o.mrani@math.ubc.ca \\ Deshin Finlay \\ Department of Mathematics, University of Michigan, \\ Ann Arbor 48109-1043, USA \\ dfinlay@umich.edu
}

Received 15 June 2017

Accepted 27 April 2018

\begin{abstract}
It is shown explicitly how one can obtain elements of Lie groups as compositions of products of other elements based on the commutator properties of associated Lie algebras. Problems of this kind can arise naturally in control theory. Suppose an apparatus has mechanisms for moving in a limited number of ways with other movements generated by compositions of allowed motions. Two concrete examples are: (1) the restricted parallel parking problem where the commutator of translations in $y$ and rotations in the $x y$-plane yields translations in $x$. Here the control problem involves a vehicle that can only perform a series of translations in $y$ and rotations with the aim of efficiently obtaining a pure translation in $x$; (2) involves an apparatus that can only perform rotations about two axes with the aim of performing rotations about a third axis. Both examples involve three-dimensional Lie algebras. In particular, the composition problem is solved for the nine threeand four-dimensional Lie algebras with non-trivial solutions. Three different solution methods are presented. Two of these methods depend on operator and matrix representations of a Lie algebra. The other method is a differential equation method that depends solely on the commutator properties of a Lie algebra. Remarkably, for these distinguished Lie algebras the solutions involve arbitrary functions and can be expressed in terms of elementary functions.
\end{abstract}

Keywords: Lie groups, Lie algebras, commutators.

\section{Introduction}

Lie groups and their representations play an important role in various applications. Lie groups of transformations describe rigid body motions (rotations and translations), scalings, as well as other transformations. In this paper it is shown explicitly how one can obtain basis elements of Lie groups as compositions of products of other basis elements, motivated by the commutator properties of associated Lie algebras. Problems of this kind can arise naturally in control theory $[3,8,9]$. Here an apparatus has mechanisms for moving in a limited number of ways and the aim is to generate efficiently other movements from compositions of possible motions. Two concrete examples are: (1) the restricted parallel parking problem where the commutator of translations in $y$ and rotations 
in the $x y$-plane yields translations in $x$. Here the control problem involves a vehicle that can only perform translations in $y$ and rotations with the aim of efficiently obtaining a pure translation in $x$; (2) involves an apparatus that can only perform rotations about two axes with the aim of generating rotations about a third axis. Here the commutator of rotations about two axes yields rotations about the third axis. Both examples involve three-dimensional Lie algebras. In terms of the notation used in [11], examples (1) and (2) respectively include the Lie algebras $S_{3,3}$ with its parameter set to zero and $s o(3, \mathbb{R})$. The authors are unaware of literature that has solved this composition problem.

Three distinct methods are presented to solve the composition problem. The first method (operator method) depends on realizing a Lie group as a Lie group of transformations. Such realizations can be found in [6] for some and in [10] for all three- and four-dimensional Lie algebras.

The second method (matrix representation method) involves matrix representations of finitedimensional Lie algebras which are known to exist from Ado's theorem [1]. This theorem states that there exists a faithful square matrix representation for every finite-dimensional Lie algebra. There are many existing algorithms that generate such matrices including one developed by Willem de Graaf [7]. While the minimal dimension of a matrix representation is not known in general, it is known for three- and four-dimensional Lie algebras (see [4] and [5]). This method is also applied to a control theory problem in [8].

The third method (DE method) was initially presented in [2] for other purposes. This method only uses the commutator properties of a Lie algebra. In particular, it does not require the use of a representation of a Lie algebra. The DE method involves setting up and solving an initial value problem for a nonlinear system of first order ordinary differential equations. The DE method yields a necessary condition for solutions - it turns out that for all three- and four-dimensional cases, the DE method yields all solutions.

Remarkably, for all relevant $n$-dimensional Lie algebras, $n=3$ or 4 , when the considered composition has $n+1$ Lie group elements, the solution involves one arbitrary function and can be expressed in terms of elementary functions.

In Section 2, we give a precise mathematical statement of the research problem. Then we describe fully the three different methods used to solve it. As an illustrative example, we focus on the Lie algebra $s l(2, F)$. In Section 3, in two tables we summarize our results for all relevant three- and four-dimensional Lie algebras. Following this, we show the essential details that yield these solutions. Finally in Section 4, we make further remarks and discuss the advantages and disadvantages of the three presented methods.

\section{Research problem}

Consider a three-dimensional Lie algebra $L$, if $B_{1}, B_{2}$, and $B_{3}$ form a basis for $L$ then the commutator of $B_{1}$ and $B_{2}$ is a linear combination of the basis elements of $L$, i.e.,

$$
\left[B_{1}, B_{2}\right]=B_{1} B_{2}-B_{2} B_{1}=\sum_{k=1}^{3} C_{12}^{k} B_{k},
$$

in terms of real structure constants $C_{12}^{1}, C_{12}^{2}$, and $C_{12}^{3}$. For the research problem under consideration we require that $C_{12}^{3} \neq 0$. In other words, the Lie algebra element $B_{3}$ is generated by the other two elements. All six three-dimensional Lie algebras presented in [11] have at least one commutator that satisfies this property. In particular, for the problem under consideration, it does not matter what the other commutators of $L$ are. The question of interest is whether the Lie group element generated by 
$B_{3}$ can be obtained from the Lie group elements generated by $B_{1}$ and $B_{2}$ as illustrated by the two examples mentioned in the introduction. Motivated by the commutator property (2.1) with $C_{12}^{3} \neq 0$, the aim is to find continuous functions $a(\varepsilon), b(\varepsilon), c(\varepsilon)$, and $d(\varepsilon)$ so that the equation

$$
e^{a(\varepsilon) B_{1}} e^{b(\varepsilon) B_{2}} e^{c(\varepsilon) c} e^{d(\varepsilon) B_{2}}=e^{\varepsilon B_{3}}
$$

with

$$
a(0)=b(0)=c(0)=d(0)=0
$$

holds for an arbitrary value of $\varepsilon$. Next, to clarify how the research problem given by (2.2) follows from (2.1) with $C_{12}^{3} \neq 0$, a concrete example is presented. where

The parallel parking problem has commutators given by $[R, Y]=X,[R, X]=-Y,[X, Y]=0$,

$$
X=-\frac{\partial}{\partial x}, \quad Y=\frac{\partial}{\partial y}, R=y \frac{\partial}{\partial x}-x \frac{\partial}{\partial y}
$$

The first commutator indicates that $X$ can be generated from $R$ and $Y$. This leads one to consider either equation

$$
e^{a(\varepsilon) R} e^{b(\varepsilon) Y} e^{c(\varepsilon) R} e^{d(\varepsilon) Y}=e^{\varepsilon X}
$$

or

$$
e^{a(\varepsilon) Y} e^{b(\varepsilon) R} e^{c(\varepsilon) Y} e^{d(\varepsilon) R}=e^{\varepsilon X} .
$$

In this example $R, Y$, and $X$ correspond to $B_{1}, B_{2}$, and $B_{3}$ respectively. The solution to equation (2.3) will be presented in Section 3. Note that when the commutator of the Lie algebras used in equation (2.2) do not satisfy the assumptions stated above, one would not expect any non-trivial solutions. For example, since in the parking problem the commutators of $X$ and $Y$ do not generate $R$, the equation

$$
e^{a(\varepsilon) X} e^{b(\varepsilon) Y} e^{c(\varepsilon) X} e^{d(\varepsilon) Y}=e^{\varepsilon R}
$$

does not have a non-trivial solution.

When the DE method is used, additional assumptions about these functions are needed. In particular, here $a(\varepsilon), b(\varepsilon), c(\varepsilon)$, and $d(\varepsilon)$ are differentiable everywhere except at $\varepsilon=0$. This assumption is needed since the DE method relies on finding a system of differential equations that the four functions must satisfy.

In general, it turns out that the problem as stated will always have a degree of freedom in its solution. Moreover, a minimum number of four terms are needed on the left hand side of equation (2.2). This follows from the origin of the commutator equation (2.1). In particular, from the form of equation (2.2), one would expect, as will be seen later in this paper, that there are solutions for which $a(\varepsilon), b(\varepsilon), c(\varepsilon)$, and $d(\varepsilon)$ are of order $\sqrt{\varepsilon}$ as $\varepsilon \rightarrow 0$ in order to generate a commutator element of order $\varepsilon$ on the right-hand side of equation (2.2).

Now consider a four-dimensional Lie algebra $L$ with basis elements $B_{1}, B_{2}, B_{3}$, and $B_{4}$ such that the elements $B_{1}, B_{2}$, and $B_{3}$ do not form a subalgebra. For the research problem we require that the 
commutator of $B_{1}$ and $B_{2}$ satisfies

$$
\left[B_{1}, B_{2}\right]=B_{1} B_{2}-B_{2} B_{1}=\sum_{k=1}^{4} C_{12}^{k} B_{k},
$$

with real structure constants $C_{12}^{1}, \ldots, C_{12}^{4}$ where $C_{12}^{3} \neq 0$ and $C_{12}^{4}=0$. Here the Lie algebra element $B_{3}$ can be generated from the elements $B_{1}$ and $B_{2}$. The problem of interest is whether the Lie group element generated by $B_{3}$ can be obtained from the Lie group elements generated by $B_{1}$ and $B_{2}$. In particular, in view of the commutator property (2.5), we are interested in finding continuous functions $a(\varepsilon), b(\varepsilon), c(\varepsilon), d(\varepsilon), f(\varepsilon)$, and $g(\varepsilon)$ so that the equation

$$
e^{a(\varepsilon) B_{1}} e^{b(\varepsilon) B_{2}} e^{c(\varepsilon) B_{1}} e^{d(\varepsilon) B_{2}} e^{f(\varepsilon) B_{1}} e^{g(\varepsilon) B_{2}}=e^{\varepsilon B_{3}},
$$

with $a(0)=b(0)=c(0)=d(0)=f(0))=g(0)=0$ holds for an arbitrary value of $\varepsilon$.

It is essential to note that the left hand side of (2.6) is composed of the product of six Lie group elements: in all considered cases there exist solutions where one of $a(\varepsilon)$ or $g(\varepsilon)$ is zero, but this is not determinate a priori.

One should also note that one can state the problems in (2.2) and (2.6) with the roles of $B_{1}$ and $B_{2}$ interchanged when the number of terms to the left of these equations is even. This does not change the nature of the problem. For the Lie algebras where both problems were considered, it was found that they led to isomorphic solutions.

In this paper, for all relevant three- and four-dimensional Lie algebras, we present three different methods that can yield the general solution for their respective equations (2.2) and (2.6). In what follows, we will describe the different methods used to solve (2.2) and (2.6). As a simple example, in this section we solve the composition problem for the three-dimensional Lie algebra $\operatorname{sl}(2, F)$ to illustrate how these different methods work. The solution of the composition problem for the other relevant three- and four-dimensional Lie algebras will be presented in Section 3.

We first note that $s l(2, F)$ has the commutators

$$
[X, Y]=Z,[Z, X]=2 X,[Y, Z]=2 Y .
$$

\subsection{Operator method}

The operator method requires a representation of a Lie algebra in terms of differential operators. The operator representation is not necessarily unique. This lack of uniqueness is illustrated by the example of $\operatorname{sl}(2, F)$.

\subsubsection{Description of the procedure for the operator method}

Let $\left\{\Delta_{1}, \ldots, \Delta_{k}\right\}$ be a differential operator representation of a Lie algebra $L$ which respectively has basis elements $\left\{B_{1}, \ldots, B_{k}\right\}$, where

$$
\Delta_{i}=\gamma_{j}^{i}(\boldsymbol{x}) \frac{\partial}{\partial x_{j}}, \quad i=1, \ldots, k, \text { and } \boldsymbol{x}=\left(x_{1}, \ldots, x_{m}\right) .
$$

Then equations (2.2) and (2.6) become respectively the equations

$$
e^{a(\varepsilon) \Delta_{1}} e^{b(\varepsilon) \Delta_{2}} e^{c(\varepsilon) \Delta_{1}} e^{d(\varepsilon) \Delta_{2}} \boldsymbol{x}=e^{\varepsilon \Delta_{3}} \boldsymbol{x},
$$


and

$$
e^{a(\varepsilon) \Delta_{1}} e^{b(\varepsilon) \Delta_{2}} e^{c(\varepsilon) \Delta_{1}} e^{d(\varepsilon) \Delta_{2}} e^{f(\varepsilon) \Delta_{1}} e^{g(\varepsilon) \Delta_{2}} x=e^{\varepsilon \Delta_{3}} x,
$$

where $e^{\varepsilon \Delta_{i}} \boldsymbol{x}=\sum_{n=0}^{\infty} \frac{\varepsilon^{n}}{n !} \Delta_{i}^{n} \boldsymbol{x}=\left(\boldsymbol{x}^{i}\right)^{*}$ for $i=1, \ldots, k ;\left(\boldsymbol{x}^{i}\right)^{*}=\left(\left(x_{1}^{i}\right)^{*}, \ldots,\left(x_{m}^{i}\right)^{*}\right)$ is the image of $\boldsymbol{x}$ with respect to the $i$ th basis element of the Lie group of transformations connected with the differential operator $\Delta_{i}, i=1, \ldots, k$.

From the First Fundamental Theorem of Lie, one can also obtain $\left(x^{i}\right)^{*}$ by solving the system of differential equations $\frac{d\left(x_{j}^{i}\right)^{*}}{d \varepsilon}=\gamma_{j}^{i}(\boldsymbol{x}), j=1, \ldots, m$, with initial condition $\left.\left(x_{j}^{i}\right)\right)^{*}(0)=x_{j}$.

\subsubsection{Example $\operatorname{sl}(2, F)$}

Operator representations for $s l(2, F)$ include

$$
\begin{gathered}
\Delta_{1}=X=-x^{2} \frac{\partial}{\partial x}, \Delta_{2}=Y=\frac{\partial}{\partial x}, \Delta_{3}=Z=2 x \frac{\partial}{\partial x}, \text { in } \mathbb{R} ; \\
\Delta_{1}=X=-y \frac{\partial}{\partial x}, \Delta_{2}=Y=-x \frac{\partial}{\partial y}, \Delta_{3}=Z=y \frac{\partial}{\partial y}-x \frac{\partial}{\partial x}, \text { in } \mathbb{R}^{2} .
\end{gathered}
$$

For the operator representation (2.10) for $s l(2, F)$, one obtains

$$
e^{\varepsilon X}(x)=\frac{x}{1+\varepsilon x}, e^{\varepsilon Y}(x)=x+\varepsilon, e^{\varepsilon Z}(x)=e^{2 \varepsilon} x .
$$

There are two well-known methods to obtain (2.12).

Method I. From the First Fundamental Theorem of Lie, the operator representation (2.10) leads to solving separately the three IVPs

$$
\begin{aligned}
& \frac{d x^{*}}{d \varepsilon}=-x^{* 2}, x^{*}(0)=x ; \\
& \frac{d x^{*}}{d \varepsilon}=1, x^{*}(0)=x ; \\
& \frac{d x^{*}}{d \varepsilon}=2 x^{*}, x^{*}(0)=x .
\end{aligned}
$$

It is easy to show that the three Lie group transformations (2.12) respectively solve the IVPs (2.13)(2.15).

Method II. Using induction, it is easy to show that

$X^{n} x=(-1)^{n} n ! x^{n+1}, n \geq 0$. Hence $e^{\varepsilon X} x=\sum_{n=0}^{\infty} \frac{\varepsilon^{n}}{n !} X^{n} x=\sum_{n=0}^{\infty} \varepsilon^{n}(-1)^{n} x^{n+1}=\frac{x}{1+\varepsilon x}$. Since $Y x=1, Y^{n} x=0$, $n \geq 2$, it follows that $e^{\varepsilon Y} x=x+\varepsilon$. It is easy to show that $Z^{n} x=2^{n} x, n \geq 0$. Hence $e^{\varepsilon Z} x=e^{2 \varepsilon} x$. 
To proceed, it is convenient to rewrite expression (2.8) in the form

$$
e^{b(\varepsilon) Y} e^{c(\varepsilon) X} e^{d(\varepsilon) Y} x=e^{-a(\varepsilon) X} e^{\varepsilon Z} x .
$$

Then from (2.12), one obtains

$$
\begin{gathered}
e^{b(\varepsilon) Y} e^{c(\varepsilon) X} e^{d(\varepsilon) Y} x=e^{b(\varepsilon) Y} e^{c(\varepsilon) X}(x+d)=e^{b(\varepsilon) Y}\left(d+\frac{x}{1+c x}\right)=d+\frac{x+b}{1+c(x+b)}, \\
e^{-a(\varepsilon) X} e^{\varepsilon Z}(x)=e^{-a(\varepsilon) X}\left(e^{2 \varepsilon} x\right)=e^{2 \varepsilon} \frac{x}{1-a x} .
\end{gathered}
$$

Then expression (2.16) becomes $d+\frac{x+b}{1+c(x+b)}=e^{2 \varepsilon} \frac{x}{1-a x}$. Hence for all $x$, one has

$$
-\left(a(d c+1)+c e^{2 \varepsilon}\right) x^{2}+\left(-a(d+b+b d c)+d c+1-e^{2 \varepsilon}(1+b c)\right) x+b+d+b d c=0 .
$$

This yields the set of equations

$$
b+d+b d c=0, d c+1=e^{2 \varepsilon}(1+b c),-a(d c+1)=c e^{2 \varepsilon} .
$$

The solution to the system of equations (2.17) is given by

$$
a(\varepsilon)=\frac{e^{\varepsilon}-e^{2 \varepsilon}}{d(\varepsilon)}, \quad b(\varepsilon)=-e^{-\varepsilon} d(\varepsilon), c(\varepsilon)=\frac{e^{\varepsilon}-1}{d(\varepsilon)},
$$

where $d(\varepsilon)$ is any continuous function chosen so that $a(\varepsilon)$ and $c(\varepsilon)$ are continuous functions, and satisfying $d(\varepsilon) \neq 0$ for any $\varepsilon \neq 0$ with $a(0)=b(0)=c(0)=d(0)=0$.

\subsection{Matrix representation method}

The matrix representation method involves a matrix representation of $L$. In particular, one seeks an appropriate matrix for each basis element of $L$ using the Lie algebra package of the computer software GAP (Group, Algorithms, Programming) [7]. A difficulty arose in the case of the fourdimensional Lie algebra $S_{4,7}$ (in terms of the nomenclature used in the classification of Lie algebras in [11]). Here the matrix representation obtained from the software package [7] could not be used since the obtained representation is not isomorphic to $S_{4,7}$. For this Lie algebra, we used a matrix representation given in [4].

\subsubsection{Description of the procedure used for the matrix representation method}

Let $L$ be a $k$-dimensional Lie algebra with basis elements $B_{i}$ with $M_{i}$ denoting a matrix representation of $B_{i}, i=1, \ldots, k$.

Step 1. Find matrices $\left\{M_{i}\right\}$ that represent $L$ using computer software or relevant literature ( [4], [7]).

Step 2. Attempt to find a closed form representation for each element $e^{\varepsilon B_{i}}$ of the Lie group associated with $L$ from the Taylor expansion $e^{\varepsilon M_{i}}=\sum_{n=0}^{\infty} \frac{\varepsilon^{n}}{n !} M_{i}^{n}$ where $M_{i}^{0}=I$ for $i=1, \ldots, k$. (In all three- and four-dimensional cases, this step was successful in leading to such a closed form representation.)

Step 3. Compute $\prod_{i=1}^{2 k-2} e^{a_{i}(\varepsilon) B_{j}}$ where $\left\{\begin{array}{l}j=1 \text { if } i \text { is odd, } \\ j=2 \text { if } i \text { is even. }\end{array}\right.$

Step 4. Solve equations (2.2) and (2.6). 


\subsubsection{Example $\operatorname{sl}(2, F)$}

Although $\operatorname{sl}(2, F)$ is a three-dimensional Lie algebra, a matrix representation is given by the $2 \times 2$ matrices $X=\left(\begin{array}{ll}0 & 1 \\ 0 & 0\end{array}\right), \quad Y=\left(\begin{array}{ll}0 & 0 \\ 1 & 0\end{array}\right), \quad Z=\left(\begin{array}{cc}1 & 0 \\ 0 & -1\end{array}\right)$. Then $X^{2}=\left(\begin{array}{ll}0 & 0 \\ 0 & 0\end{array}\right)$. Hence $X^{n}=\left(\begin{array}{ll}0 & 0 \\ 0 & 0\end{array}\right), n \geq 2$. Thus $e^{\varepsilon X}=I+\varepsilon X=\left(\begin{array}{ll}1 & \varepsilon \\ 0 & 1\end{array}\right)$. Similarly, one can show that $e^{\varepsilon Y}=\left(\begin{array}{ll}1 & 0 \\ \varepsilon & 1\end{array}\right)$. One can easily show that $Z^{n}=$ $\left(\begin{array}{cc}1 & 0 \\ 0 & (-1)^{n}\end{array}\right)$. Hence $e^{\varepsilon Z}=\left(\begin{array}{cc}e^{\varepsilon} & 0 \\ 0 & e^{-\varepsilon}\end{array}\right)$. Consequently,

$$
M=e^{a(\epsilon) X} e^{b(\epsilon) Y} e^{c(\epsilon) X} e^{d(\epsilon) Y}=\left(\begin{array}{cc}
a b c d+a b+a d+c d+1 & a+c+a b c \\
b+d+b c d & b c+1
\end{array}\right) .
$$

After setting $M=e^{\varepsilon Z}$, one obtains the equations

$$
b c+1=e^{-\varepsilon}, \quad b+d+b c d=0, a+c+a b c=0, a b c d+a b+a d+c d+1=e^{\varepsilon},
$$

whose solution is given by (2.18).

\subsection{DE method}

The DE method requires differentiation of the unknown functions in equations (2.2) and (2.6). It involves setting up a nonlinear system of first order ordinary differential equations that must be satisfied by all differentiable solutions of equations (2.2) and (2.6). Here the solutions respectively satisfy initial conditions

$$
\begin{gathered}
a(0)=b(0)=c(0)=d(0)=0, \\
a(0)=b(0)=c(0)=d(0)=f(0)=g(0)=0
\end{gathered}
$$

in the three- and four-dimensional cases. Note that when $\varepsilon=0$, the unknown functions will be continuous but not differentiable. The DE method yields a necessary condition for solutions. Sufficiency is shown from the solutions obtained by the other two methods.

\subsubsection{Description of the procedure used for the DE method}

After differentiating equations (2.2) and (2.6) with respect to $\varepsilon$, one obtains respectively,

$$
\begin{gathered}
a_{1}^{\prime}(\varepsilon) B_{1} \prod_{i=1}^{4} e^{a_{i} B_{i}}+a_{2}^{\prime}(\varepsilon) e^{a_{1} B_{1}} B_{2} \prod_{i=2}^{4} e^{a_{i} B_{i}}+a_{3}^{\prime}(\varepsilon) \prod_{i=1}^{2} e^{a_{i} B_{i}} B_{1} \prod_{i=3}^{4} e^{a_{i} B_{i}} \\
+a_{4}^{\prime}(\varepsilon) \prod_{i=1}^{3} e^{a_{i} B_{i}} B_{2} e^{a_{4} B_{2}}=B_{3} e^{\varepsilon B_{3}}, \\
a_{1}^{\prime}(\varepsilon) B_{1} \prod_{i=1}^{6} e^{a_{i} B_{i}}+a_{2}^{\prime}(\varepsilon) e^{a_{1} B_{1}} B_{2} \prod_{i=2}^{6} e^{a_{i} B_{i}}+\cdots+a_{6}^{\prime}(\varepsilon) \prod_{i=1}^{5} e^{a_{i} B_{i}} B_{2} e^{a_{6} B_{2}}=B_{3} e^{\varepsilon B_{3}},
\end{gathered}
$$

where $B_{i}=\left\{\begin{array}{l}B_{1} \text { if } i \text { is odd } \\ B_{2} \text { if } i \text { is even }\end{array}\right.$ and $a_{1}=a, a_{2}=b, a_{3}=c, a_{4}=d, a_{5}=f, a_{6}=g$.

From equations (2.21) and (2.22), one sees that a formula is needed for pulling the products of the 
exponentials appropriately to the right of each $B_{p}$ in order to get back $\prod_{i=1}^{n} e^{a_{i}(\varepsilon) B_{i}}=e^{\varepsilon B_{3}}, n=4,6$, respectively.

In general, one proceeds as follows.

Step 1 . Find $\left\{f_{j}\right\}$ so that

$$
e^{\varepsilon B_{i}} B_{p}=\sum_{j=1}^{k} f_{j} B_{j} e^{\varepsilon B_{i}}
$$

where $i=1,2, p=1, \ldots, k$, and $k$ is the dimension of the Lie algebra $L$. Since Ado's theorem [7] guarantees the existence of a matrix representation for every finite-dimensional Lie algebra, one can treat the operations in $L$ as matrix elements so that, without loss of generality, $L$ is associative.

Step 2. Differentiate equations (2.2) and (2.6) with respect to $\varepsilon$ to obtain equations (2.21) and (2.22), respectively. Then appropriately and recursively substitute equation (2.23) into equations (2.21) and (2.22). Thus in each case one obtains an equation of the form

$$
\sum_{j=1}^{k} \alpha_{j}(\varepsilon) B_{j} \prod_{i=1}^{k} e^{a_{i} B_{i}}=B_{3} e^{\varepsilon B_{3}},
$$

for specific functions $\alpha_{j}(\varepsilon)$.

Step 3. Assume that expressions (2.2) and (2.6) hold. Consequently, this yields necessary conditions that $\left\{\alpha_{j}(\varepsilon)\right\}$ must satisfy, namely the nonlinear system of first order ODEs

$$
\begin{aligned}
& \alpha_{3}(\varepsilon)=1, \\
& \alpha_{i}(\varepsilon)=0, \quad i \neq 3,
\end{aligned}
$$

with initial conditions (2.19) and (2.20), respectively.

Step 4. Check that the solution of the ODE system (2.25) solves respectively expressions (2.2) or (2.6) using either the matrix or operator method.

\subsubsection{Example $\operatorname{sl}(2, F)$}

Theorem 2.1. For $s l(2, F)$ these identities hold for any $\varepsilon$.

$$
\begin{aligned}
& e^{\varepsilon X} Y \equiv\left(Y+\varepsilon Z-\varepsilon^{2} X\right) e^{\varepsilon X}, e^{\varepsilon X} Z \equiv(Z-2 \varepsilon X) e^{\varepsilon X}, e^{\varepsilon Y} X \equiv\left(X-\varepsilon Z-\varepsilon^{2} Y\right) e^{\varepsilon Y}, \\
& e^{\varepsilon Y} Z=(Z+2 \varepsilon Y) e^{\varepsilon Y} .
\end{aligned}
$$

Proof. From the commutator relations (2.7), one directly obtains

$$
\begin{aligned}
& X Y=Y X+Z, \\
& X Z=Z X-2 X, \\
& Y Z=Z Y+2 Y
\end{aligned}
$$

Hence $X^{2} Y=Y X^{2}+2 Z X-2 X$. Then it is easy to show that $X^{n} Y=Y X^{n}+n Z X^{n-1}-n(n-1) X^{n-1}, n \in \mathbb{N}$. 
Similarly, one can show that the following relations hold.

$$
\begin{aligned}
& X^{n} Z=Z X^{n}-2 n X^{n}, \\
& Y^{n} X=X Y^{n}-n Z Y^{n-1}-n(n-1) Y^{n-1}, \\
& Y^{n} Z=Z Y^{n}+2 n Y^{n} .
\end{aligned}
$$

Consequently,

$$
e^{\varepsilon X} Y=Y e^{\varepsilon X}+\varepsilon Z \sum_{n=1}^{\infty} \frac{\varepsilon^{n-1}}{(n-1) !} X^{n-1}-\varepsilon^{2} X \sum_{n=2}^{\infty} \frac{\varepsilon^{n-2}}{(n-2) !} X^{n-2}=\left(Y+\varepsilon Z-\varepsilon^{2} X\right) e^{\varepsilon X} .
$$

Similarly, one obtains the remaining relations in (2.26), completing the proof.

Now to proceed further, we differentiate equation (2.6) with respect to $\varepsilon$. This yields

$$
\begin{aligned}
& a^{\prime}(\varepsilon) X e^{\varepsilon Z}+b^{\prime}(\varepsilon) e^{a(\varepsilon) X} Y e^{b(\varepsilon) Y} e^{c(\varepsilon) X} e^{d(\varepsilon) Y}+c^{\prime}(\varepsilon) e^{a(\varepsilon) X} e^{b(\varepsilon) Y} X e^{c(\varepsilon) X} e^{d(\varepsilon) Y} \\
& +d^{\prime}(\varepsilon) e^{a(\varepsilon) X} e^{b(\varepsilon) Y} e^{c(\varepsilon) X} Y e^{d(\varepsilon) Y}=Z e^{\varepsilon Z} .
\end{aligned}
$$

Using the relations in Theorem 2.1, one finds that equation (2.27) becomes $\left[\alpha_{1}(\varepsilon) X+\alpha_{2}(\varepsilon) Y+\alpha_{3}(\varepsilon) Z\right] e^{\varepsilon Z}=Z e^{\varepsilon Z}$ with

$$
\begin{aligned}
& \alpha_{1}(\varepsilon)=a^{\prime}-a^{2}\left(b^{\prime}+d^{\prime}+b\left(-b c^{\prime}+b c^{2} d^{\prime}+2 c d^{\prime}\right)\right)+c^{\prime}+2 a b c^{\prime}-2 a c d^{\prime}-c^{2} d^{\prime}-2 a b c^{2} d^{\prime}=0, \\
& \alpha_{2}(\varepsilon)=b^{\prime}+d^{\prime}+b\left(-b c^{\prime}+b c^{2} d^{\prime}+2 c d^{\prime}\right)=0 \\
& \alpha_{3}(\varepsilon)=a\left(b^{\prime}+d^{\prime}+b\left(-b c^{\prime}+b c^{2} d^{\prime}+2 c d^{\prime}\right)\right)-b c^{\prime}+c d^{\prime}+b c^{2} d^{\prime}=1 .
\end{aligned}
$$

After substituting equation (2.29) into equations (2.28) and (2.30), one gets respectively

$$
\begin{gathered}
a^{\prime}+c^{\prime}-c^{2} d^{\prime}-2 a\left(-b c^{\prime}+c d^{\prime}+b c^{2} d^{\prime}\right)=0, \\
-b c^{\prime}+c d^{\prime}+b c^{2} d^{\prime}=1 .
\end{gathered}
$$

After substituting equation (2.32) into each of equations (2.31) and (2.29), one obtains

$$
\begin{aligned}
& a^{\prime}+c^{\prime}-c^{2} d^{\prime}-2 a=0, \\
& b^{\prime}+d^{\prime}+b c d^{\prime}+b=0 .
\end{aligned}
$$

After subtracting $c$ times equation (2.34) from equation (2.32), one finds that $(b c)^{\prime}+b c=-1$. Hence

$$
b(\varepsilon)=\frac{e^{-\varepsilon}-1}{c(\varepsilon)} .
$$

Substitution of equation (2.35) into equation (2.34) leads to $d^{\prime}=\frac{c e^{\varepsilon}-c^{\prime}\left(e^{\varepsilon}-1\right)}{c^{2}}$. Consequently,

$$
d(\varepsilon)=\frac{e^{\varepsilon}-1}{c(\varepsilon)} .
$$

After substituting equation (2.36) into equation (2.33), one gets $2\left(a+e^{\varepsilon} c\right)=\left(a+e^{\varepsilon} c\right)^{\prime}$. Hence $a(\varepsilon)=-c(\varepsilon) e^{\varepsilon}$. 
Thus the solution to the system of differential equations (2.25)-(2.27) with initial condition (2.16) is given by (2.18).

\section{Results}

Using the procedures described in Section 2, the results for all relevant three- and four-dimensional Lie algebras are presented in Tables 1 and 2, respectively.

Table 1: Results for three-dimensional Lie algebras

\begin{tabular}{|c|c|c|}
\hline $\begin{array}{l}\text { Lie algebra; } \\
\text { commutators }\end{array}$ & Composition equation & Solution \\
\hline $\begin{array}{l}s l(2, F) \\
{[X, Y]=Z} \\
{[X, Z]=-2 X} \\
{[Y, Z]=2 Y}\end{array}$ & $e^{a(\varepsilon) X} e^{b(\varepsilon) Y} e^{c(\varepsilon) X} e^{d(\varepsilon) Y}=e^{\varepsilon Z}$ & $\begin{array}{l}d(\varepsilon) \text { is an arbitrary function } \\
\text { satisfying } d(\varepsilon) \neq 0 \text { when } \varepsilon \neq 0 \\
a(\varepsilon)=\frac{e^{\varepsilon}-e^{\varepsilon \varepsilon}}{d(\varepsilon)} \\
b(\varepsilon)=\frac{e^{-\varepsilon}-1}{e^{\varepsilon}-1} d(\varepsilon) \\
c(\varepsilon)=\frac{e^{\varepsilon}-1}{d(\varepsilon)}\end{array}$ \\
\hline $\begin{array}{l}\text { Parallel parking } \\
\text { problem, } \\
S_{3,3 \text { with }} \\
\text { constant } r=0 \\
{[R, Y]=X} \\
{[R, X]=-Y} \\
{[X, Y]=0}\end{array}$ & $e^{a(\varepsilon) R} e^{b(\varepsilon) Y} e^{c(\varepsilon) R} e^{d(\varepsilon) Y}=e^{\varepsilon X}$ & $\begin{array}{l}c(\varepsilon) \text { is an arbitrary function } \\
\text { satisfying } c(\varepsilon) \neq k \pi \text { for every } \\
k \in \mathbb{Z} \text { when } \varepsilon \neq 0 \\
a(\varepsilon)=-c(\varepsilon) \\
b(\varepsilon)=-\varepsilon \csc c(\varepsilon) \\
d(\varepsilon)=\varepsilon \cot c(\varepsilon)\end{array}$ \\
\hline $\begin{array}{l}\text { Euler angles } \\
\text { problem, } \\
\text { so( }(3, \mathbb{R}) \\
{[X, Y]=Z} \\
{[X, Z]=-Y} \\
{[Y, Z]=X}\end{array}$ & $e^{a(\varepsilon) X} e^{b(\varepsilon) Y} e^{c(\varepsilon) X} e^{d(\varepsilon) Y}=e^{\varepsilon Z}$ & $\begin{array}{l}\text { Any } c(\varepsilon) \text { satisfying } c(\varepsilon) \neq k \pi \text { for } \\
\text { every } k \in \mathbb{Z} \text { when } \varepsilon \neq 0 \text { and } \\
\left|\frac{\sin \varepsilon}{\sin c(\varepsilon)}\right| \leq 1 \text { with } \\
a(\varepsilon)=-\arccos \left(\frac{\cos c(\varepsilon)}{\cos \varepsilon}\right) \\
b(\varepsilon)=-\arcsin \left(\frac{\sin \varepsilon}{\sin c(\varepsilon)}\right) \\
d(\varepsilon)=\arccos \left(-\frac{\sin a(\varepsilon)}{\sin c(\varepsilon)}\right) \\
a(\varepsilon)=\arccos \left(\frac{\cos c(\varepsilon)}{\cos \varepsilon}\right) \\
b(\varepsilon)=\pi+\arcsin \left(\frac{\sin \varepsilon}{\sin c(\varepsilon)}\right) \\
d(\varepsilon)=\arccos \left(-\frac{\sin a(\varepsilon)}{\sin c(\varepsilon)}\right)\end{array}$ \\
\hline $\begin{array}{l}n_{3,1} \\
{[X, Y]=Z} \\
{[X, Z]=0} \\
{[Z, Y]=0}\end{array}$ & $e^{a(\varepsilon) X} e^{b(\varepsilon) Y} e^{c(\varepsilon) X} e^{d(\varepsilon) Y}=e^{\varepsilon Z}$ & $\begin{array}{l}d(\varepsilon) \text { is an arbitrary function } \\
\text { satisfying } d(\varepsilon) \neq 0 \text { when } \varepsilon \neq 0 \\
a(\varepsilon)=-\frac{\varepsilon}{d(\varepsilon)} \\
b(\varepsilon)=-d(\varepsilon) \\
c(\varepsilon)=\frac{\varepsilon}{d(\varepsilon)}\end{array}$ \\
\hline
\end{tabular}


G.W. Bluman et al. / Composition of Lie Group Elements from Basis Lie Algebra Elements

Table 1 - continued from previous page

\begin{tabular}{|l|l|l|}
\hline $\begin{array}{l}\text { Lie algebra; } \\
\text { commutators }\end{array}$ & \multicolumn{1}{|c|}{ Composition equation } & \multicolumn{1}{c|}{ Solution } \\
\hline$S_{3,1}$ & & \\
{$[Y, Z]=-Y$} & & $d(\varepsilon)$ is an arbitrary function \\
{$[Y, X]=0$} & satisfying $d(\varepsilon) \neq 0$ when $\varepsilon \neq 0$ \\
{$[Z, X]=r X+Y$} & $e^{a(\varepsilon) X} e^{b(\varepsilon) Z} e^{c(\varepsilon) X} e^{d(\varepsilon) Z}=e^{\varepsilon Y}$ & $b(\varepsilon)=-d(\varepsilon)$ \\
where $r$ is a & & $a(\varepsilon)=\frac{\varepsilon(1-r)}{1-e^{(r-1) d(\varepsilon)}}$ \\
constant & & $c(\varepsilon)=-a(\varepsilon) e^{r d(\varepsilon)}$ \\
satisfying $|r| \leq 1$ & & \\
\hline$S_{3,2}$ & & $d(\varepsilon)$ is an arbitrary function \\
{$[Z, X]=X$} & & satisfying $d(\varepsilon) \neq 0$ when $\varepsilon \neq 0$ \\
{$[Z, Y]=X+Y$} & $e^{a(\varepsilon) Y} e^{b(\varepsilon) Z} e^{c(\varepsilon) Y} e^{d(\varepsilon) Z}=e^{\varepsilon X}$ & $b(\varepsilon)=-d(\varepsilon)$ \\
{$[X, Y]=0$} & & $c(\varepsilon)=-\frac{\varepsilon e^{d(\varepsilon)}}{d(\varepsilon)}$ \\
& & $a(\varepsilon)=\frac{\varepsilon}{d(\varepsilon)}$ \\
\hline$S_{3,3}$ general & & $c(\varepsilon)$ is an arbitrary function \\
case & & satisfying $c(\varepsilon) \neq k \pi$ for every \\
{$[R, X]=r X-Y$} & & $k \in \mathbb{Z}$ when $\varepsilon \neq 0$ \\
{$[R, Y]=X+r Y$} & $e^{a(\varepsilon) R} e^{b(\varepsilon) Y} e^{c(\varepsilon) R} e^{d(\varepsilon) Y}=e^{\varepsilon X}$ & $a(\varepsilon)=-c(\varepsilon)$ \\
{$[X, Y]=0$} & & $b(\varepsilon)=-\varepsilon \csc c(\varepsilon) e^{r c(\varepsilon)}$ \\
where $r$ is a & & $d(\varepsilon)=\varepsilon \cot c(\varepsilon)$ \\
non-negative & & \\
constant. & & \\
\hline
\end{tabular}

Table 2: Results for four-dimensional Lie algebras

\begin{tabular}{|l|l|l|}
\hline $\begin{array}{l}\text { Lie algebra; } \\
\text { commutators }\end{array}$ & \multicolumn{1}{|c|}{ Composition equation } & \multicolumn{1}{|c|}{ Solution } \\
\hline$S_{4,2}$ & & $c(\varepsilon)$ is an arbitrary function \\
{$[W, X]=X$} & satisfying $c(\varepsilon) \neq 0$ when \\
& & \\
{$[W, Y]=X+Y$} & & $a(\varepsilon)=f(\varepsilon)=-\frac{1}{2} c(\varepsilon)$ \\
{$[W, Z]=Y+Z$} & $e^{a(\varepsilon) W} e^{b(\varepsilon) Z} e^{c(\varepsilon) W} e^{d(\varepsilon) Z} e^{f(\varepsilon) W}=e^{\varepsilon Y}$ & $b(\varepsilon)=-\frac{\varepsilon}{c(\varepsilon)} e^{\frac{1}{2} c(\varepsilon)}$ \\
{$[X, Y]=0$} & & $d(\varepsilon)=\frac{\varepsilon}{c(\varepsilon)} e^{-\frac{1}{2} c(\varepsilon)}$ \\
{$[X, Z]=0$} & & \multicolumn{1}{|c|}{ Continued on next page } \\
{$[Z, Y]=0$} & & \multicolumn{1}{|c}{} \\
\hline \multicolumn{2}{|c|}{} \\
\hline
\end{tabular}


Table 2 - continued from previous page

\begin{tabular}{|c|c|c|}
\hline $\begin{array}{l}\text { Lie algebra; } \\
\text { commutators }\end{array}$ & Composition equation & Solution \\
\hline $\begin{array}{l}S_{4,7} \\
{[Y, Z]=X} \\
{[W, Y]=-Z} \\
{[W, Z]=Y} \\
{[W, X]=0} \\
{[X, Y]=0} \\
{[X, Z]=0}\end{array}$ & $e^{a(\varepsilon) Z} e^{b(\varepsilon) W} e^{c(\varepsilon) Z} e^{d(\varepsilon) W} e^{f(\varepsilon) Z}=e^{\varepsilon Y}$ & $\begin{array}{l}b(\varepsilon) \text { is an arbitrary function } \\
\text { satisfying } b(\varepsilon) \neq k \pi \text { for every } \\
k \in \mathbb{Z} \text { when } \varepsilon \neq 0 \\
a(\varepsilon)=-\frac{\varepsilon}{2 \tan b(\varepsilon)} \\
c(\varepsilon)=\frac{\varepsilon}{\sin b(\varepsilon)} \\
d(\varepsilon)=-b(\varepsilon) \\
f(\varepsilon)=-\frac{\varepsilon}{2 \tan b(\varepsilon)}\end{array}$ \\
\hline $\begin{array}{l}S_{4,9} \\
{[Y, Z]=X} \\
{[W, Y]=r Y-Z} \\
{[W, Z]=Y+r Z} \\
{[W, X]=2 r X} \\
{[X, Y]=0} \\
{[X, Z]=0}\end{array}$ & $e^{a(\varepsilon) W} e^{b(\varepsilon) Z} e^{c(\varepsilon) W} e^{d(\varepsilon) Z} e^{f(\varepsilon) W}=e^{\varepsilon Y}$ & $\begin{array}{l}b(\varepsilon) \text { is an arbitrary function } \\
\text { satisfying } b(\varepsilon) \neq k \pi \text { for every } \\
k \in \mathbb{Z} \text { when } \varepsilon \neq 0 \\
a(\varepsilon)=-\frac{\varepsilon}{2 \tan b(\varepsilon)} \\
c(\varepsilon)=\frac{\varepsilon e^{-r b}}{\sin b(\varepsilon)} \\
d(\varepsilon)=-b(\varepsilon) \\
f(\varepsilon)=-\frac{\varepsilon}{2 \tan b(\varepsilon)}\end{array}$ \\
\hline $\begin{array}{l}S_{4,10} \\
{[Y, Z]=X} \\
{[W, Y]=Y} \\
{[W, Z]=Y+Z} \\
{[W, X]=2 X} \\
{[X, Y]=0} \\
{[X, Z]=0}\end{array}$ & $\begin{array}{l}e^{a(\varepsilon) W} e^{b(\varepsilon) Z} e^{c(\varepsilon) W} e^{d(\varepsilon) Z} e^{f(\varepsilon) W} e^{g(\varepsilon) Z}= \\
e^{\varepsilon Y}\end{array}$ & $\begin{array}{l}a(\varepsilon) \text { and } c(\varepsilon) \text { are arbitrary } \\
\text { functions satisfying } \\
a(\varepsilon) c(\varepsilon) \neq 0, a(\varepsilon)+c(\varepsilon) \neq 0, \\
\text { and } c(\varepsilon)^{2}+a(\varepsilon) c(\varepsilon) \geq 0 \\
f(\varepsilon)=-(a(\varepsilon)+c(\varepsilon)) \\
b(\varepsilon)= \\
\varepsilon\left(\frac{c(\varepsilon) \pm \sqrt{c(\varepsilon)^{2}+a(\varepsilon) c(\varepsilon)}}{a(\varepsilon) c(\varepsilon)}\right) e^{-a(\varepsilon)} \\
g(\varepsilon)=-\frac{\varepsilon+b(\varepsilon) c(\varepsilon) e^{a(\varepsilon)}}{a(\varepsilon)+c(\varepsilon)} \\
d(\varepsilon)= \\
-g(\varepsilon) e^{-(a(\varepsilon)+c(\varepsilon))}-b(\varepsilon) e^{-c(\varepsilon)} \\
\text { and in the limiting case when } \\
a(\varepsilon)=0, \\
f(\varepsilon)=-c(\varepsilon) \\
d(\varepsilon)=\frac{\varepsilon e^{-c(\varepsilon)}}{c(\varepsilon)} \\
b(\varepsilon)=g(\varepsilon)=-\frac{\varepsilon}{2 c(\varepsilon)}\end{array}$ \\
\hline
\end{tabular}

The sketch of the proofs of the results, exhibited in Tables 1 and 2, follow.

\subsection{Parallel parking problem (Lie algebra $S_{3,3}$ with constant $r=0$ )}

\subsubsection{Model example}

To illustrate parallel parking, as an example, consider a unicycle that performs forward and backward translations as well as rotations. The unicycle is represented by a straight line with centre 
located at $(x, y)$ and initial orientation parallel to the $y$-axis with its centre located at $(0,0)$ in Figure 1 . The aim is to move the unicycle so that its centre finishes at $(\varepsilon, 0)$ with the vehicle parallel to its initial orientation by a succession of rotations and translations in the same direction as the straight line. As will be illustrated in Figure 1, the minimum number of steps that start with a nonzero translation is four. Let $d(\varepsilon)$ and $b(\varepsilon)$ be the translations in the first and third steps, respectively and let $c(\varepsilon)$ and $a(\varepsilon)$ be the angles of rotation in the second and last steps, respectively. Since the direction the vehicle is facing at the end must be the same as at the start, one must have $a(\varepsilon)=-c(\varepsilon)$. From Figure 1, one sees that $d(\varepsilon)+b(\varepsilon) \cos c(\varepsilon)=0$ and $b(\varepsilon) \sin c(\varepsilon)=\varepsilon$. Hence the solution to this problem is given by

$$
a(\varepsilon)=-c(\varepsilon), \quad b(\varepsilon)=\frac{\varepsilon}{\sin c(\varepsilon)}, d(\varepsilon)=-\varepsilon \cot c(\varepsilon),
$$

where $c(\varepsilon)$ is any continuous function chosen so that $b(\varepsilon)$ and $d(\varepsilon)$ are continuous functions, and satisfying $c(\varepsilon) \neq k \pi$ for every $k \in \mathbb{Z}$ and $\varepsilon \neq 0$ with $a(0)=b(0)=c(0)=d(0)=0$.

Alternatively, one could treat $d(\varepsilon)$ as an arbitrary function. Here the solution as reflected by Figure 1 is given by

$$
a(\varepsilon)=-c(\varepsilon), \quad c(\varepsilon)=-\arctan \left(\frac{\varepsilon}{d}\right),|b(\varepsilon)|=\sqrt{d^{2}+\varepsilon^{2}},
$$

where $d(\varepsilon)$ is any continuous function chosen so that $c(\varepsilon)$ is a continuous function, and satisfying $d(\varepsilon) \neq 0$ and $-\frac{2}{\pi \varepsilon}<d(\varepsilon)<\frac{2}{\pi \varepsilon}$ for every $\varepsilon \neq 0$ with $a(0)=b(0)=c(0)=d(0)=0$.

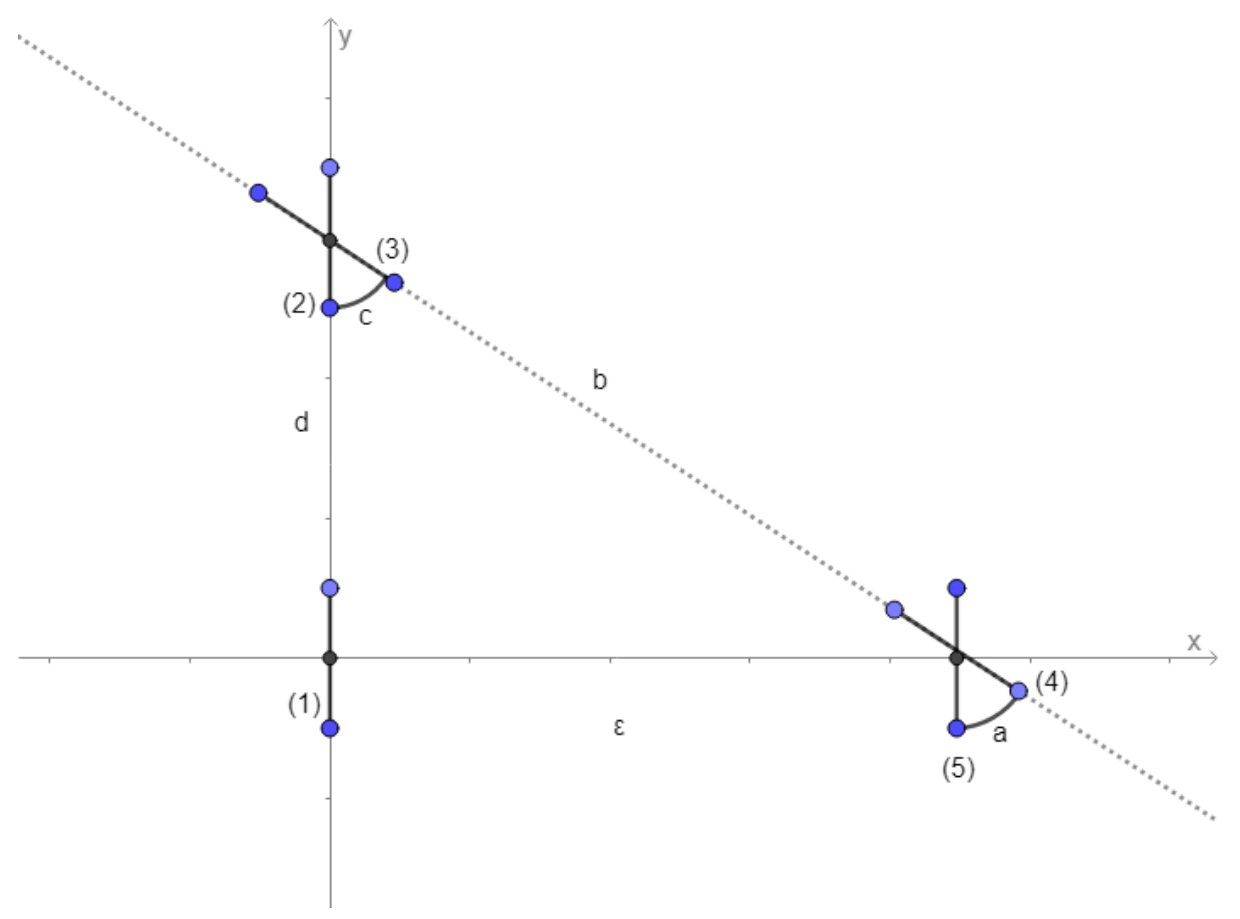

Fig. 1: Illustration of solution of parallel parking problem. (1) represents initial configuration of vehicle with successive configurations represented by (2) to (5). 
G.W. Bluman et al. / Composition of Lie Group Elements from Basis Lie Algebra Elements

Next, the solution to the parallel parking problem is presented using the methods described in Section 2 which can be applied to any Lie algebra.

\subsubsection{Solution using the operator method}

An operator representation for this Lie algebra is given by

$$
X=-\frac{\partial}{\partial x}, \quad Y=\frac{\partial}{\partial y}, R=y \frac{\partial}{\partial x}-x \frac{\partial}{\partial y} .
$$

Consequently,

$$
\begin{aligned}
M & =e^{a(\varepsilon) R} e^{b(\varepsilon) Y} e^{c(\varepsilon) R} e^{d(\varepsilon) Y}(x, y)=e^{a(\varepsilon) R} e^{b(\varepsilon) Y} e^{c(\varepsilon) R}(x, y+d) \\
& =e^{a(\varepsilon) R} e^{b(\varepsilon) Y}(x \cos c+y \sin c, d+y \cos c-x \sin c) \\
& =e^{a(\varepsilon) R}(b \sin c+x \cos c+y \sin c, d+b \cos c+y \cos c-x \sin c) \\
& =(b \sin c+x \cos (a+c)+y \sin (a+c), d+b \cos c-x \sin (a+c)+y \cos (a+c)) .
\end{aligned}
$$

Then $M=e^{\varepsilon X}(x, y)=(x-\varepsilon, y)$ iff

$$
\sin (a+c)=0, \quad \cos (a+c)=1, d+b \cos c=0,-b \sin c=\varepsilon .
$$

The solution to the system of equations (3.2) is given by (3.1).

\subsubsection{Solution using the matrix representation method}

A matrix representation of $S_{3,3}$ with constant $r=0$ is given by

$$
X=\left(\begin{array}{lll}
0 & 0 & 1 \\
0 & 0 & 0 \\
0 & 0 & 0
\end{array}\right), \quad Y=\left(\begin{array}{ccc}
0 & 0 & 0 \\
0 & 0 & -1 \\
0 & 0 & 0
\end{array}\right), R=\left(\begin{array}{ccc}
0 & -1 & 0 \\
1 & 0 & 0 \\
0 & 0 & 0
\end{array}\right) .
$$

Hence $X^{n}=Y^{n}=0, n \geq 2$.

Thus $e^{\varepsilon Y}=I+\varepsilon Y=\left(\begin{array}{ccc}1 & 0 & 0 \\ 0 & 1 & -\varepsilon \\ 0 & 0 & 1\end{array}\right)$, and $e^{\varepsilon X}=\left(\begin{array}{lll}1 & 0 & \varepsilon \\ 0 & 1 & 0 \\ 0 & 0 & 1\end{array}\right)$.

For all $k>0$, one has

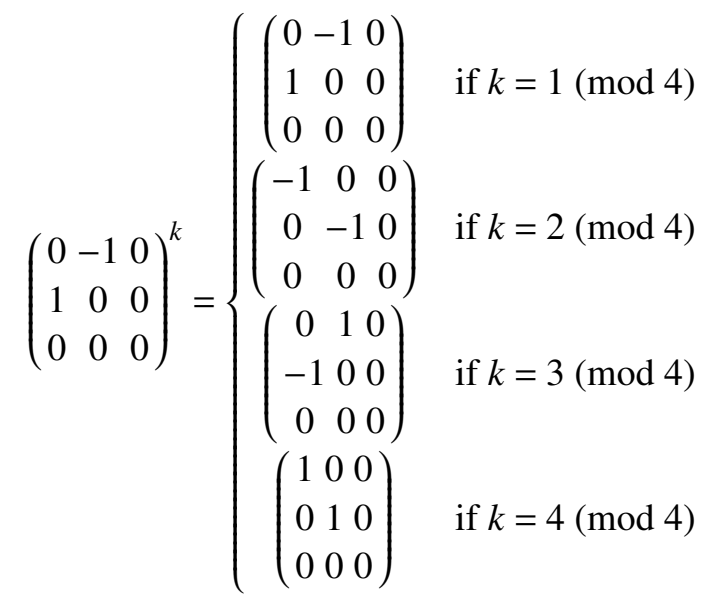


Consequently,

$$
e^{\varepsilon R}=\left(\begin{array}{ccc}
\cos \varepsilon-\sin \varepsilon & 0 \\
\sin \varepsilon & \cos \varepsilon & 0 \\
0 & 0 & 1
\end{array}\right)
$$

Hence

$$
M=e^{a(\varepsilon) R} e^{b(\varepsilon) Y} e^{c(\varepsilon) R} e^{d(\varepsilon) Y}=\left(\begin{array}{ccc}
\cos (a+c)-\sin (a+c) & d \sin (a+c)+b \sin a \\
\sin (a+c) & \cos (a+c) & -d \cos (a+c)-b \cos a \\
0 & 0 & 1
\end{array}\right) .
$$

Then $M=e^{\varepsilon X}$ is satisfied iff

$$
\cos (a+c)=1, \quad \sin (a+c)=0, \quad b \sin a=\varepsilon, d+b \cos a=0 .
$$

The solution to the system of equations (3.3) is given by (3.1).

\subsubsection{Solution using the DE method}

For all $n \geq 0$, one can show that

$$
\begin{aligned}
& X^{n} Y=Y X^{n}, \quad X^{n} R=R X^{n}-n Y X^{n-1}, \\
& R^{n} X=X \sum_{\substack{i=0 \\
i \text { even }}}^{n}(-1)^{\frac{i}{2}}\left(\begin{array}{c}
n \\
i
\end{array}\right) R^{n-i}-Y \sum_{\substack{i=1 \\
i \text { odd }}}^{n}(-1)^{\frac{i+1}{2}}\left(\begin{array}{c}
n \\
i
\end{array}\right) R^{n-i}, \\
& R^{n} Y=Y \sum_{\substack{i=0 \\
i \text { even }}}^{n}(-1)^{\frac{i}{2}}\left(\begin{array}{c}
n \\
i
\end{array}\right) R^{n-i}+X \sum_{\substack{i=1 \\
i \text { odd }}}^{n}(-1)^{\frac{i+1}{2}}\left(\begin{array}{c}
n \\
i
\end{array}\right) R^{n-i} .
\end{aligned}
$$

Using these results, one can easily obtain the identities

$$
\begin{aligned}
& e^{\varepsilon Y} X \equiv X e^{\varepsilon Y}, e^{\varepsilon Y} R \equiv(R-\varepsilon X) e^{\varepsilon Y}, e^{\varepsilon R} X \equiv(\cos \varepsilon X-\sin \varepsilon Y) e^{\varepsilon R}, \\
& e^{\varepsilon R} Y \equiv(\cos \varepsilon Y+\sin \varepsilon X) e^{\varepsilon R} .
\end{aligned}
$$

Now to proceed, we differentiate with respect to $\varepsilon$ the equation

$$
e^{a(\varepsilon) R} e^{b(\varepsilon) Y} e^{c(\varepsilon) R} e^{d(\varepsilon) Y}=e^{\varepsilon X} .
$$

Thus

$$
\begin{aligned}
& a^{\prime} R e^{a(\varepsilon) R} e^{b(\varepsilon) Y} e^{c(\varepsilon) R} e^{d(\varepsilon) Y}+b^{\prime} e^{a(\varepsilon) R} Y e^{b(\varepsilon) Y} e^{c(\varepsilon) R} e^{d(\varepsilon) Y}+c^{\prime} e^{a(\varepsilon) R} e^{b(\varepsilon) Y} R e^{c(\varepsilon) R} e^{d(\varepsilon) Y}+ \\
& d^{\prime} e^{a(\varepsilon) R} e^{b(\varepsilon) Y} e^{c(\varepsilon) R} Y e^{d(\varepsilon) Y}=X e^{\varepsilon X} .
\end{aligned}
$$

Using the identities in (3.5), one can show that equation (3.7) leads to the ODE system

$$
\begin{aligned}
& a^{\prime}+c^{\prime}=0, \quad \cos a b^{\prime}+b \sin a c^{\prime}+\cos (a+c) d^{\prime}=0, \\
& \sin a b^{\prime}-b \cos a c^{\prime}+\sin (a+c) d^{\prime}=1 .
\end{aligned}
$$

It is easy to show that the solution to the ODE system (3.8) is given by (3.1). 


\subsection{Euler angles problem (Lie algebra so $(3, \mathbb{R})$ )}

\subsubsection{Solution using the operator method}

An operator representation for this Lie algebra is given by

$$
X=x \frac{\partial}{\partial y}-y \frac{\partial}{\partial x}, \quad Y=y \frac{\partial}{\partial z}-z \frac{\partial}{\partial y}, Z=x \frac{\partial}{\partial z}-z \frac{\partial}{\partial x} .
$$

Here setting $e^{a(\varepsilon) X} e^{b(\varepsilon) Y} e^{c(\varepsilon) X} e^{d(\varepsilon) Y}=e^{\varepsilon Z}$ leads to the system of nine equations

$$
\begin{aligned}
& \sin c \sin b=-\sin \varepsilon, \quad \cos c-\cos a \cos \varepsilon=0, \quad \sin c \cos b+\sin a \cos \varepsilon=0, \\
& \cos d \sin c+\sin a=0, \cos b \cos c \cos d-\sin b \sin d=\cos a, \\
& \sin b \cos c \cos d+\cos b \sin d=0, \sin b \cos d+\cos b \cos c \sin d=\sin a \sin \varepsilon, \\
& \sin c \sin d-\cos a \sin \varepsilon=0, \cos b \cos d-\sin b \cos c \sin d=\cos \varepsilon,
\end{aligned}
$$

whose solutions are given by

$$
\begin{aligned}
& a(\varepsilon)=-\arccos \left(\frac{\cos c(\varepsilon)}{\cos \varepsilon}\right), b(\varepsilon)=-\arcsin \left(\frac{\sin \varepsilon}{\sin c(\varepsilon)}\right), d(\varepsilon)=\arccos \left(-\frac{\sin a(\varepsilon)}{\sin c(\varepsilon)}\right) \\
& a(\varepsilon)=\arccos \left(\frac{\cos c(\varepsilon)}{\cos \varepsilon}\right), b(\varepsilon)=\pi+\arcsin \left(\frac{\sin \varepsilon}{\sin c(\varepsilon)}\right), d(\varepsilon)=\arccos \left(-\frac{\sin a(\varepsilon)}{\sin c(\varepsilon)}\right) .
\end{aligned}
$$

In solution (3.10), for any $\varepsilon \neq 0, c(\varepsilon)$ is any continuous function chosen so that $a(\varepsilon)$ and $d(\varepsilon)$ are continuous, and satisfying $\left|\frac{\sin \varepsilon}{\sin c(\varepsilon)}\right| \leq 1$ with $c(\varepsilon) \neq k \pi$ for every $k$ in $\mathbb{Z}$ and such that $a(0)=b(0)=$ $c(0)=d(0)=0$.

\subsubsection{Solution using the matrix representation method}

A matrix representation of $s o(3, \mathbb{R})$ is given by

$$
X=\left(\begin{array}{ccc}
0 & 0 & 0 \\
0 & 0 & -1 \\
0 & 1 & 0
\end{array}\right), \quad Y=\left(\begin{array}{ccc}
0 & 0 & 1 \\
0 & 0 & 0 \\
-1 & 0 & 0
\end{array}\right), \quad Z=\left(\begin{array}{ccc}
0 & -1 & 0 \\
1 & 0 & 0 \\
0 & 0 & 0
\end{array}\right)
$$

One can show that

$$
e^{\varepsilon X}=\left(\begin{array}{ccc}
1 & 0 & 0 \\
0 & \cos \varepsilon-\sin \varepsilon \\
0 & \sin \varepsilon & \cos \varepsilon
\end{array}\right), \quad e^{\varepsilon Y}=\left(\begin{array}{ccc}
\cos \varepsilon & 0 & \sin \varepsilon \\
0 & 1 & 0 \\
-\sin \varepsilon & 0 & \cos \varepsilon
\end{array}\right), \quad e^{\varepsilon Z}=\left(\begin{array}{ccc}
\cos \varepsilon-\sin \varepsilon & 0 \\
\sin \varepsilon & \cos \varepsilon & 0 \\
0 & 0 & 1
\end{array}\right)
$$


Consequently, one can show that the entries $\left\{a_{i j}\right\}$ of the matrix $M=e^{a(\varepsilon) X} e^{b(\varepsilon) Y} e^{c(\varepsilon) X} e^{d(\varepsilon) Y}$ are given by

$$
\begin{aligned}
& a_{11}=\cos b \cos d-\sin b \cos c \sin d, a_{12}=\sin b \sin c, \\
& a_{13}=\cos b \sin d+\sin b \cos c \cos d, \\
& a_{21}=\sin a \sin b \cos d+\cos a \sin c \sin d+\sin a \cos b \cos c \sin d, \\
& a_{22}=\cos a \cos c-\sin a \cos b \sin c, \\
& a_{23}=\sin a \sin b \sin d-\cos a \sin c \cos d-\sin a \cos b \cos c \cos d, \\
& a_{31}=-\cos a \sin b \cos d+\sin a \sin c \sin d-\cos a \cos b \cos c \sin a, \\
& a_{32}=\sin a \cos c+\sin c \cos a \cos b, \\
& a_{33}=-\sin d \cos a \sin b-\sin a \sin c \cos d+\cos a \cos b \cos c \cos d .
\end{aligned}
$$

From the matrix equation $M=e^{\varepsilon Z}$, one obtains a system of equations that can be simplified to (3.9). Hence the solutions are given by (3.10) and (3.11).

\subsubsection{Solution using the DE method}

One can show the following identities hold for all $\varepsilon$.

$$
\begin{aligned}
& e^{\varepsilon X} Y \equiv(\cos \varepsilon Y+\sin \varepsilon Z) e^{\varepsilon X}, e^{\varepsilon X} Z \equiv(\cos \varepsilon Z-\sin \varepsilon Y) e^{\varepsilon X}, \\
& e^{\varepsilon Y} X \equiv(\cos \varepsilon X-\sin \varepsilon Z) e^{\varepsilon Y}, e^{\varepsilon Y} Z \equiv(\cos \varepsilon Z+\sin \varepsilon X) e^{\varepsilon Y} .
\end{aligned}
$$

After differentiating with respect to $\varepsilon$ the equation $e^{a(\varepsilon) X} e^{b(\varepsilon) Y} e^{c(\varepsilon) X} e^{d(\varepsilon) Y}=e^{\varepsilon Z}$ and using the identities in (3.12), one obtains the simplified ODE system

$$
\cos b a^{\prime}+c^{\prime}=-\sin b \cos a, \quad \sin b a^{\prime}+\sin c d^{\prime}=\cos a \cos b, \quad b^{\prime}+\cos c d^{\prime}=\sin a .
$$

The ODE system (3.13) admits (3.10) and (3.11) as solutions.

\subsection{Lie algebra $n_{3,1}$}

\subsubsection{Solution using the operator method}

From [10], an operator representation for $n_{3,1}$ is given by $X=\frac{\partial}{\partial x}, Y=x \frac{\partial}{\partial z}, Z=\frac{\partial}{\partial z}$.

Consequently, equation $e^{a(\varepsilon) X} e^{b(\varepsilon) Y} e^{c(\varepsilon) X} e^{d(\varepsilon) Y}(x, z)=e^{\varepsilon Z}(x, z)$ leads to the equation

$$
(x+a+c,(b+d)(x+a)+d c+z)=(x, z+\varepsilon) .
$$

It is easy to see that the solution to equation (3.14) is given by

$$
a(\varepsilon)=-\frac{\varepsilon}{d(\varepsilon)}, \quad b(\varepsilon)=-d(\varepsilon), \quad c(\varepsilon)=\frac{\varepsilon}{d(\varepsilon)},
$$

where $d(\varepsilon)$ is any continuous function chosen so that $a(\varepsilon)$ and $c(\varepsilon)$ are continuous functions, and satisfying $d(\varepsilon) \neq 0$ for any $\varepsilon \neq 0$ with $a(0)=b(0)=c(0)=d(0)=0$. 


\subsubsection{Solution using the matrix representation method}

From [7], a matrix representation of $n_{3,1}$ is given by

$$
X=\left(\begin{array}{lll}
0 & 1 & 0 \\
0 & 0 & 0 \\
0 & 0 & 0
\end{array}\right), Y=\left(\begin{array}{lll}
0 & 0 & 0 \\
0 & 0 & 1 \\
0 & 0 & 0
\end{array}\right), Z=\left(\begin{array}{lll}
0 & 0 & 1 \\
0 & 0 & 0 \\
0 & 0 & 0
\end{array}\right) .
$$

Then one can show that

$$
e^{\varepsilon X}=\left(\begin{array}{lll}
1 & \varepsilon & 0 \\
0 & 1 & 0 \\
0 & 0 & 1
\end{array}\right), e^{\varepsilon Y}=\left(\begin{array}{lll}
1 & 0 & 0 \\
0 & 1 & \varepsilon \\
0 & 0 & 1
\end{array}\right), e^{\varepsilon Z}=\left(\begin{array}{lll}
1 & 0 & \varepsilon \\
0 & 1 & 0 \\
0 & 0 & 1
\end{array}\right) .
$$

Accordingly, one can show that

$$
M=e^{a(\varepsilon) X} e^{b(\varepsilon) Y} e^{c(\varepsilon) X} e^{d(\varepsilon) Y}=\left(\begin{array}{ccc}
1 & a+c c d+a d+a b \\
0 & 1 & b+d \\
0 & 0 & 1
\end{array}\right) .
$$

Consequently $M=e^{\varepsilon Z}$ yields the system of equations

$$
a+c=0, \quad b+d=0, c d+a d+a b=\varepsilon,
$$

whose solution is given by (3.15).

\subsubsection{Solution using the DE method}

One can readily obtain the following identities which hold for all $\varepsilon$.

$$
e^{\varepsilon X} Y \equiv(X+\varepsilon Z) e^{\varepsilon X}, e^{\varepsilon X} Z \equiv Z e^{\varepsilon X}, e^{\varepsilon Y} X \equiv(Y-\varepsilon Z) e^{\varepsilon Y}, e^{\varepsilon Y} Z \equiv Z e^{\varepsilon Y} .
$$

After differentiating with respect to $\varepsilon$ the equation $e^{a(\varepsilon) X} e^{b(\varepsilon) Y} e^{c(\varepsilon) X} e^{d(\varepsilon) Z}=e^{\varepsilon Z}$ and using the identities (3.16), one obtains the ODE system $a^{\prime}+c^{\prime}=0, b^{\prime}+d^{\prime}=0, a b^{\prime}-b c^{\prime}+(a+c) d^{\prime}=1$, whose solution is given by (3.15).

\subsection{Lie algebra $S_{3,1}$}

\subsubsection{Solution using the operator method}

An operator representation [10] for $S_{3,1}$ is given by

$$
X=\frac{\partial}{\partial x}+\frac{\partial}{\partial y}, \quad Y=(1-r) \frac{\partial}{\partial x}, Z=-x \frac{\partial}{\partial x}-r y \frac{\partial}{\partial y} .
$$

Consequently, the equation $e^{a(\varepsilon) X} e^{b(\varepsilon) Z} e^{c(\varepsilon) X} e^{d(\varepsilon) Z}(x, y)=e^{\varepsilon Y}(x, y)$ leads to the equation

$$
\left(e^{-(b+d)}\left(x+a+c e^{b}\right), e^{-r(b+d)}\left(y+a+c e^{r b}\right)\right)=((1-r) \varepsilon+x, y) .
$$

It is easy to see that equation (3.17) is satisfied iff

$$
b=-d, a+c e^{-d}=(1-r) \varepsilon, a+c e^{-r d}=0 .
$$


The solution to the system of equations (3.18) is given by

$$
a(\varepsilon)=\frac{\varepsilon(1-r)}{1-e^{(r-1) d(\varepsilon)}}, \quad b(\varepsilon)=-d(\varepsilon), \quad c(\varepsilon)=-a(\varepsilon) e^{r d(\varepsilon)},
$$

where $d(\varepsilon)$ is any continuous function chosen so that $a(\varepsilon)$ is a continuous function, and satisfying $d(\varepsilon) \neq 0$ for any $\varepsilon \neq 0$ with $a(0)=b(0)=c(0)=d(0)=0$.

Note that in the limiting case where $r \rightarrow 1$, equation (3.19) becomes $a(\varepsilon)=-\frac{\varepsilon}{d(\varepsilon)}, b(\varepsilon)=-d(\varepsilon)$, and $c(\varepsilon)=-a(\varepsilon) e^{d(\varepsilon)}$.

\subsubsection{Solution using the matrix representation method}

A matrix representation of $S_{3,1}$ is given by

$$
X=\left(\begin{array}{ccc}
0 & -r & 0 \\
0 & 0 & 0 \\
0 & -1 & 0
\end{array}\right), Y=\left(\begin{array}{ccc}
0 & 0 & 0 \\
0 & 0 & 0 \\
0 & -1 & 0
\end{array}\right), Z=\left(\begin{array}{lll}
r & 0 & 0 \\
0 & 0 & 0 \\
1 & 0 & 1
\end{array}\right)
$$

Then one can easily show that

$$
e^{\varepsilon X}=\left(\begin{array}{ccc}
1 & -r \varepsilon & 0 \\
0 & 1 & 0 \\
0 & -\varepsilon & 1
\end{array}\right), \quad e^{\varepsilon Y}=\left(\begin{array}{ccc}
1 & 0 & 0 \\
0 & 1 & 0 \\
0 & -\varepsilon & 1
\end{array}\right), e^{\varepsilon Z}=\left(\begin{array}{ccc}
e^{\varepsilon r} & 0 & 0 \\
0 & 1 & 0 \\
\frac{e^{\varepsilon}-e^{\varepsilon r}}{1-r} & 0 & e^{\varepsilon}
\end{array}\right)
$$

Hence

$$
M=e^{a(\varepsilon) X} e^{b(\varepsilon) Z} e^{c(\varepsilon) X} e^{d(\varepsilon) Z}=\left(\begin{array}{ccc}
e^{r(b+d)} & -r a-c r e^{b r} & 0 \\
0 & 1 & 0 \\
\frac{e^{b+d}-e^{r(b+d)}}{1-r} & \frac{c r\left(e^{b}-e^{b r}\right)}{r-1}-a-c e^{b} & e^{b+d}
\end{array}\right) .
$$

Then equation $M=e^{\varepsilon Y}$ leads to the system of equations

$$
a+c e^{b r}=0, \quad b+d=0, \frac{c r\left(e^{b}-e^{b r}\right)}{1-r}+a+c e^{b}=\varepsilon,
$$

with solution given by (3.19).

\subsubsection{Solution using the DE method}

One can show that the following identities hold for all $\varepsilon$.

$$
e^{\varepsilon X} Y \equiv Y e^{\varepsilon X}, e^{\varepsilon X} Z \equiv(Z-r \varepsilon X-\varepsilon Y) e^{\varepsilon X}, e^{\varepsilon Z} X \equiv\left(e^{\varepsilon r} X+\frac{e^{\varepsilon}-e^{\varepsilon r}}{1-r} Y\right) e^{\varepsilon Z}, e^{\varepsilon Z} Y \equiv(Y+\varepsilon Y) e^{\varepsilon Z}
$$

After differentiating with respect to $\varepsilon$ the equation $e^{a(\varepsilon) X} e^{b(\varepsilon) Z} e^{c(\varepsilon) X} e^{d(\varepsilon) Z}=e^{\varepsilon Y}$ and using the identities (3.21), one obtains the ODE system

$$
a^{\prime}-r a b^{\prime}+e^{r b} c^{\prime}-r\left(a+c e^{r b}\right) d^{\prime}=0, \quad b^{\prime}+d^{\prime}=0, \frac{e^{b}-e^{r b}}{1-r}\left(c^{\prime}-r c d^{\prime}\right)-c e^{b} d^{\prime}=1,
$$

whose solution is given by (3.19). 


\subsection{Lie algebra $S_{3,2}$}

\subsubsection{Solution using the operator method}

From [10], an operator representation for $S_{3,2}$ is given by $X=\frac{\partial}{\partial x}, Y=y \frac{\partial}{\partial x}, Z=-x \frac{\partial}{\partial x}+\frac{\partial}{\partial y}$.

Consequently, equation $e^{a(\varepsilon) Y} e^{b(\varepsilon) Z} e^{c(\varepsilon) Y} e^{d(\varepsilon) Z}(x, y)=e^{\varepsilon X}(x, y)$ leads to equation

$$
\left(e^{-(b+d)} x+e^{-(b+d)}\left(a+c e^{b}\right) y+b c e^{-d}, y+b+d\right)=(x+\varepsilon, y) .
$$

It is easy to see that equation (3.23) is satisfied iff

$$
b=-d, a+c e^{-d}=0, b c e^{-d}=\varepsilon .
$$

The solution to the system of equations (3.24) is given by

$$
a(\varepsilon)=\frac{\varepsilon}{d(\varepsilon)}, \quad b(\varepsilon)=-d(\varepsilon) c(\varepsilon)=-\frac{\varepsilon e^{d(\varepsilon)}}{d(\varepsilon)},
$$

where $d(\varepsilon)$ is any continuous function chosen so that $a(\varepsilon)$ and $c(\varepsilon)$ are continuous functions, and satisfying $d(\varepsilon) \neq 0$ for any $\varepsilon \neq 0$ with $a(0)=b(0)=c(0)=d(0)=0$.

\subsubsection{Solution using the matrix representation method}

A matrix representation for $S_{3,2}$ is given by

$$
X=\left(\begin{array}{ccc}
0 & 0 & -1 \\
0 & 0 & 0 \\
0 & 0 & 0
\end{array}\right), \quad Y=\left(\begin{array}{ccc}
0 & 0 & -1 \\
0 & 0 & -1 \\
0 & 0 & 0
\end{array}\right), Z=\left(\begin{array}{lll}
1 & 1 & 0 \\
0 & 1 & 0 \\
0 & 0 & 0
\end{array}\right)
$$

Then one can easily show that

$$
e^{\varepsilon X}=\left(\begin{array}{ccc}
1 & 0 & -\varepsilon \\
0 & 1 & 0 \\
0 & 0 & 1
\end{array}\right), e^{\varepsilon Y}=\left(\begin{array}{ccc}
1 & 0 & -\varepsilon \\
0 & 1 & -\varepsilon \\
0 & 0 & 1
\end{array}\right), e^{\varepsilon Z}=\left(\begin{array}{ccc}
e^{\varepsilon} & \varepsilon e^{\varepsilon} & 0 \\
0 & e^{\varepsilon} & 0 \\
0 & 0 & 1
\end{array}\right)
$$

Hence

$$
M=e^{a(\varepsilon) Y} e^{b(\varepsilon) Z} e^{c(\varepsilon) Y} e^{d(\varepsilon) Z}=\left(\begin{array}{ccc}
e^{b+d} & e^{b+d}(b+d)-c e^{b}-a-b c e^{b} \\
0 & e^{b+d} & -c e^{b}-a \\
0 & 0 & 1
\end{array}\right)
$$

Consequently, the equation $M=e^{\varepsilon X}$ leads to the system of equations

$$
c e^{b}+a=0, \quad b+d=0, c e^{b}+a+b c e^{b}=\varepsilon,
$$

whose solution is given by (3.25). 


\subsubsection{Solution using the DE method}

One can show that the following identities hold for all $\varepsilon$.

$$
e^{\varepsilon Y} X \equiv X e^{\varepsilon Y}, e^{\varepsilon Y} Z \equiv(Z-\varepsilon X-\varepsilon Y) e^{\varepsilon Y}, e^{\varepsilon Z} X \equiv\left(e^{\varepsilon} X\right) e^{\varepsilon Z}, e^{\varepsilon Z} Y \equiv\left(e^{\varepsilon} Y+\varepsilon e^{\varepsilon} X\right) e^{\varepsilon Z} .
$$

The differentiation with respect to $\varepsilon$ of the equation $e^{a(\varepsilon) Y} e^{b(\varepsilon) Z} e^{c(\varepsilon) Y} e^{d(\varepsilon) Z}=e^{\varepsilon X}$ and the repeated use of the identities (3.26) leads to the ODE system

$$
a^{\prime}-a b^{\prime}+e^{b} c^{\prime}-\left(a+c e^{b}\right) d^{\prime}=0, \quad b^{\prime}+d^{\prime}=0,-a b^{\prime}+b e^{b} c^{\prime}-\left(a+c b e^{b}\right) d^{\prime}=1,
$$

whose solution is given by (3.25).

\subsection{Lie algebra $S_{3,3}$}

\subsubsection{Solution using the operator method}

From [6] and [10], an operator representation for $S_{3,3}$ is given by

$$
X=-\frac{\partial}{\partial x}, \quad Y=-\frac{\partial}{\partial y}, R=(r x+y) \frac{\partial}{\partial x}+(r y-x) \frac{\partial}{\partial y} .
$$

Consequently, equation $e^{a(\varepsilon) R} e^{b(\varepsilon) Y} e^{c(\varepsilon) R} e^{d(\varepsilon) Y}(x, y)=e^{\varepsilon X}(x, y)$ leads to equation

$$
\begin{aligned}
& \left(\cos (a+c) e^{-r(a+c)} x-\sin (a+c) e^{-r(a+c)} y+b \sin c e^{-r c}, \cos (a+c) e^{-r(a+c)} y\right. \\
& \left.+\sin (a+c) e^{-r(a+c)} x-d-b \cos c e^{-r c}\right)=(x-\varepsilon, y) .
\end{aligned}
$$

It is easy to see that equation (3.27) is satisfied iff

$$
\sin (a+c) e^{-r(a+c)}=0, \quad \cos (a+c) e^{-r(a+c)}=1, d+b \cos c e^{-r c}=0, \quad b \sin c e^{-r c}=-\varepsilon .
$$

The solution to the system of equations (3.28) is given by

$$
a=-c(\varepsilon), \quad b=-\frac{\varepsilon e^{r c(\varepsilon)}}{\sin c(\varepsilon)}, \quad d=\frac{\varepsilon}{\tan c(\varepsilon)},
$$

where $c(\varepsilon)$ is any continuous function chosen so that $b(\varepsilon)$ and $d(\varepsilon)$ are continuous functions, and satisfying $c(\varepsilon) \neq k \pi$ for every $k \in \mathbb{Z}$ and for any $\varepsilon \neq 0$ with $a(0)=b(0)=c(0)=d(0)=0$.

\subsubsection{Solution using the matrix representation method}

A matrix representation of $S_{3,3}$ is given by

$$
X=\left(\begin{array}{ccc}
0 & 0 & 1 \\
0 & 0 & -r \\
0 & 0 & 0
\end{array}\right), Y=\left(\begin{array}{ccc}
0 & 0 & -r \\
0 & 0 & -1 \\
0 & 0 & 0
\end{array}\right), R=\left(\begin{array}{ccc}
r & -1 & 0 \\
1 & r & 0 \\
0 & 0 & 0
\end{array}\right) .
$$

Hence one can show that

$$
e^{\varepsilon X}=\left(\begin{array}{ccc}
1 & 0 & \varepsilon \\
0 & 1 & -r \varepsilon \\
0 & 0 & 1
\end{array}\right), \quad e^{\varepsilon Y}=\left(\begin{array}{ccc}
1 & 0 & -r \varepsilon \\
0 & 1 & -\varepsilon \\
0 & 0 & 1
\end{array}\right), \quad e^{\varepsilon R}=\left(\begin{array}{ccc}
\cos \varepsilon e^{r \varepsilon} & -\sin \varepsilon e^{r \varepsilon} & 0 \\
\sin \varepsilon e^{r \varepsilon} & \cos \varepsilon e^{r \varepsilon} & 0 \\
0 & 0 & 1
\end{array}\right) .
$$


Consequently, the entries $\left\{a_{i j}\right\}$ of the matrix $M=e^{a(\varepsilon) R} e^{b(\varepsilon) Y} e^{c(\varepsilon) R} e^{d(\varepsilon) Y}$ are given by

$$
\begin{aligned}
& a_{11}=a_{22}=\cos (a+c) e^{r(a+c)}, a_{12}=-\sin (a+c) e^{r(a+c)}, a_{21}=\sin (a+c) e^{r(a+c)}, \\
& a_{13}=d(-r \cos (a+c)+\sin (a+c)) e^{r(a+c)}+b(-r \cos a+\sin a) e^{r a}, \\
& a_{23}=-d(\cos (a+c)+r \sin (a+c)) e^{r(a+c)}-b(\cos a+r \sin a) e^{r a}, \\
& a_{31}=a_{32}=0, a_{33}=1 .
\end{aligned}
$$

Thus the relation $M=e^{\varepsilon X}$ yields the equations

$$
\begin{aligned}
& \cos (a+c) e^{r(a+c)}=1, \quad \sin (a+c) e^{r(a+c)}=0, \\
& d(-r \cos (a+c)+\sin (a+c)) e^{r(a+c)}+b(-r \cos a+\sin a) e^{r a}=\varepsilon, \\
& d(\cos (a+c)+r \sin (a+c)) e^{r(a+c)}+b(\cos a+r \sin a) e^{r a}=r \varepsilon,
\end{aligned}
$$

whose solution is given by (3.29).

\subsubsection{Solution using the DE method}

One can show that the following identities hold for all $\varepsilon$.

$$
\begin{aligned}
e^{\varepsilon Y} X & \equiv X e^{\varepsilon Y}, e^{\varepsilon Y} R \equiv(R-\varepsilon X-r \varepsilon Y) e^{\varepsilon Y}, e^{\varepsilon R} X \equiv e^{r \varepsilon}(\cos \varepsilon X-\sin \varepsilon Y) e^{\varepsilon R}, \\
e^{\varepsilon R} Y & \equiv e^{r \varepsilon}(\cos \varepsilon Y+\sin \varepsilon X) e^{\varepsilon R} .
\end{aligned}
$$

To proceed, one differentiates with respect to $\varepsilon$ the equation $e^{a(\varepsilon) R} e^{b(\varepsilon) Y} e^{c(\varepsilon) R} e^{d(\varepsilon) Y}=e^{\varepsilon X}$ and then uses the identities (3.30) recursively. This yields the ODE system

$$
\begin{aligned}
& a^{\prime}+c^{\prime}=0, e^{r a}\left(\cos a b^{\prime}+(b \sin a-r b \cos a) c^{\prime}+\cos (a+c) e^{r c} d^{\prime}\right)=0, \\
& e^{r a}\left(\sin a b^{\prime}-(r b \sin a+b \cos a) c^{\prime}+\sin (a+c) e^{r c} d^{\prime}\right)=1 .
\end{aligned}
$$

It is easy to show that the solution to the ODE system (3.31) is given by (3.29).

\subsection{Lie algebra $S_{4,2}$}

\subsubsection{Solution using the operator method}

From [10], an operator representation for $S_{4,2}$ is given by

$$
W=-x \frac{\partial}{\partial x}+\frac{\partial}{\partial y}+y \frac{\partial}{\partial z}, \quad X=\frac{\partial}{\partial x}, \quad Y=y \frac{\partial}{\partial x}, Z=z \frac{\partial}{\partial x} .
$$

Consequently, equation $e^{a(\varepsilon) W} e^{b(\varepsilon) Z} e^{c(\varepsilon) W} e^{d(\varepsilon) Z} e^{f(\varepsilon) W}(x, y, z)=e^{\varepsilon Y}(x, y, z)$ leads to

$$
\begin{aligned}
& \left(\left(c d+a d+a b e^{-c}\right) e^{-f} y+\left(\left(d+b e^{-c}\right) e^{-f}\right) z+e^{-(a+f+c)} x+\left(a c d+\frac{1}{2} c^{2} d\right) e^{-f}, y+a+\right. \\
& \left.c+f,(a+f+c) y+z+\frac{1}{2}(a+f+c)^{2}\right)=(x+\varepsilon y, y, z) .
\end{aligned}
$$

It is easy to see that equation (3.32) is satisfied iff

$$
a+f+c=0, d+b e^{-c}=0, c d e^{-f}=\varepsilon, 2 a+c=0 .
$$

The solution to the system of equations (3.33) is given by

$$
a(\varepsilon)=f(\varepsilon)=-\frac{1}{2} c(\varepsilon), \quad b(\varepsilon)=-\frac{\varepsilon}{c(\varepsilon)} e^{\frac{1}{2} c(\varepsilon)}, d(\varepsilon)=\frac{\varepsilon}{c(\varepsilon)} e^{-\frac{1}{2} c(\varepsilon)},
$$


where $c(\varepsilon)$ is any continuous function chosen so that $b(\varepsilon)$ and $d(\varepsilon)$ are continuous functions, and satisfying $c(\varepsilon) \neq 0$ for any $\varepsilon \neq 0$ with $a(0)=b(0)=c(0)=d(0)=f(0)=0$.

\subsubsection{Solution using the matrix representation method}

A matrix representation for $S_{4,2}$ is given by

$$
X=\left(\begin{array}{cccc}
0 & 0 & 0 & -1 \\
0 & 0 & 0 & 0 \\
0 & 0 & 0 & 0 \\
0 & 0 & 0 & 0
\end{array}\right), \quad Y=\left(\begin{array}{cccc}
0 & 0 & 0 & -1 \\
0 & 0 & 0 & -1 \\
0 & 0 & 0 & 0 \\
0 & 0 & 0 & 0
\end{array}\right), \quad Z=\left(\begin{array}{cccc}
0 & 0 & 0 & 0 \\
0 & 0 & 0 & -1 \\
0 & 0 & 0 & -1 \\
0 & 0 & 0 & 0
\end{array}\right), \quad W=\left(\begin{array}{llll}
1 & 1 & 0 & 0 \\
0 & 1 & 1 & 0 \\
0 & 0 & 1 & 0 \\
0 & 0 & 0 & 0
\end{array}\right) .
$$

One can show that

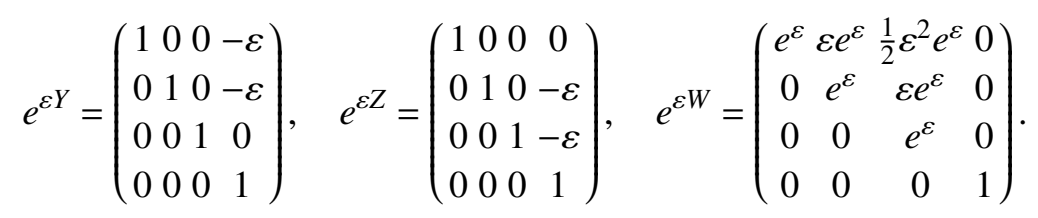

Consequently, the entries $\left\{a_{i j}\right\}$ of the matrix $M=e^{a(\epsilon) W} e^{b(\epsilon) Z} e^{c(\epsilon) W} e^{d(\epsilon) Z} e^{f(\varepsilon) W}$ are given by

$$
\begin{aligned}
& a_{11}=a_{22}=a_{33}=e^{a+c+f}, a_{12}=a_{23}=(a+c+f) e^{a+c+f}, \\
& a_{13}=\left(a f+f c+a c+\frac{1}{2}\left(a^{2}+f^{2}+c^{2}\right)\right) e^{a+c+f}, \\
& a_{14}=-d\left(a+c+a c+\frac{1}{2}\left(a^{2}+c^{2}\right)\right) e^{a+c}-b a\left(1+\frac{1}{2} a\right) e^{a}, \\
& a_{24}=-d(1+c+a) e^{a+c}-b(1+a) e^{a}, \\
& a_{34}=-d e^{a+c}-b e^{a}, a_{44}=1, a_{i j}=0 \text { for } j<i .
\end{aligned}
$$

After simplification, the relation $M=e^{\varepsilon Y}$ leads to the system of equations

$$
a+c+f=0, d\left(a+c+a c+\frac{1}{2}\left(a^{2}+c^{2}\right)\right) e^{a+c}+b a\left(1+\frac{1}{2} a\right) e^{a}=\varepsilon,
$$

$d(1+a+c) e^{a+c}+b(1+a) e^{a}=\varepsilon, d e^{a+c}+b e^{a}=0$, whose solution is given by (3.34).

\subsubsection{Solution using the DE method}

One can show that the following identities hold for all $\varepsilon$.

$$
\begin{aligned}
& e^{\varepsilon W} X \equiv e^{\varepsilon} X e^{\varepsilon W}, e^{\varepsilon W} Y \equiv\left(e^{\varepsilon} Y+\varepsilon e^{\varepsilon} X\right) e^{\varepsilon W}, e^{\varepsilon W} Z \equiv\left(e^{\varepsilon} Z+\varepsilon e^{\varepsilon} Y+\frac{1}{2} \varepsilon^{2} e^{\varepsilon} X\right) e^{\varepsilon W}, \\
& e^{\varepsilon Z} X \equiv X e^{\varepsilon Z}, e^{\varepsilon Z} Y \equiv Y e^{\varepsilon Z}, e^{\varepsilon Z} W \equiv(W-\varepsilon Z-\varepsilon Y) e^{\varepsilon Z} .
\end{aligned}
$$

After differentiating with respect to $\varepsilon$ the equation $e^{a(\varepsilon) W} e^{b(\varepsilon) Z} e^{c(\varepsilon) W} e^{d(\varepsilon) Z} e^{f(\varepsilon) W}=e^{\varepsilon Y}$ and using the identities (3.35), one obtains the system of differential equations

$$
\begin{aligned}
& a^{\prime}+c^{\prime}+f^{\prime}=0, \\
& a^{2} e^{a} b^{\prime}-b a(2+a) e^{a} c^{\prime}+(a+c)^{2} e^{a+c} d^{\prime}-\left(2 a b+2(a+c) d e^{c}+b a^{2}+d(a+c)^{2} e^{c}\right) e^{a} f^{\prime}=0, \\
& e^{a} b^{\prime}-b e^{a} c^{\prime}+e^{a+c} d^{\prime}-\left(b e^{a}+d e^{a+c}\right) f^{\prime}=0, \\
& a e^{a} b^{\prime}-b(1+a) e^{a} c^{\prime}+(a+c) e^{a+c} d^{\prime}-\left(b(1+a)+d(1+a+c) e^{c}\right) e^{a} f^{\prime}=1 .
\end{aligned}
$$

The solution to the ODE system (3.36) is given by (3.34). 


\subsection{Lie algebra $S_{4,7}$}

\subsubsection{Solution using the operator method}

From [10], an operator representation for $S_{4,7}$ is given by

$$
W=\frac{1}{2}\left(y^{2}-z^{2}\right) \frac{\partial}{\partial x}-z \frac{\partial}{\partial y}+y \frac{\partial}{\partial z}, \quad X=\frac{\partial}{\partial x}, \quad Y=\frac{\partial}{\partial y}, Z=y \frac{\partial}{\partial x}+\frac{\partial}{\partial z} .
$$

One can show that for all $\varepsilon$ the following relations hold.

$$
\begin{aligned}
e^{\varepsilon Y}(x, y, z)= & (x, y+\varepsilon, z), e^{\varepsilon Z}(x, y, z)=(x+\varepsilon y, y, z+\varepsilon), \\
e^{\varepsilon W}(x, y, z)= & \left(\frac{1}{4}\left(\sin (2 \varepsilon)\left(y^{2}-z^{2}\right)+2 \cos (2 \varepsilon) z y\right)+x-\frac{1}{2} z y, \cos \varepsilon y-\sin \varepsilon z, \cos \varepsilon z\right. \\
& +\sin \varepsilon y) .
\end{aligned}
$$

After much calculation and the use of the relations above, one can show that the equation $e^{a(\varepsilon) Z} e^{b(\varepsilon) W} e^{c(\varepsilon) Z} e^{d(\varepsilon) W} e^{f(\varepsilon) Z}(x, y, z)=e^{\varepsilon Y}(x, y, z)$ leads to the simplified system of equations

$$
f+c=0, d \sin f+\varepsilon=0, \quad b+g+d \cos f=0, g \sin f-\frac{1}{2} \varepsilon \cos f=0 .
$$

The solution to the system of equations (3.37) is given by

$$
a(\varepsilon)=f(\varepsilon)=-\frac{\varepsilon}{2 \tan b(\varepsilon)}, c(\varepsilon)=\frac{\varepsilon}{\sin b(\varepsilon)}, d(\varepsilon)=-b(\varepsilon)
$$

where $b(\varepsilon)$ is any continuous function chosen so that $a(\varepsilon), f(\varepsilon)$ and $c(\varepsilon)$ are continuous functions, and satisfying $b(\varepsilon) \neq 0$ for every $\varepsilon \neq 0$ with $a(0)=b(0)=c(0)=d(0)=f(0)=0$.

\subsubsection{Solution using the matrix representation method}

The following matrix representation for $S_{4,7}$ was found after correction of the matrix representation of $S_{4,7}$ given in [6].

$$
e^{\varepsilon Y}=\left(\begin{array}{cccc}
1 & 0 & -\varepsilon & 0 \\
0 & 1 & 0 & -\varepsilon \\
0 & 0 & 1 & 0 \\
0 & 0 & 0 & 1
\end{array}\right), \quad e^{\varepsilon Z}=\left(\begin{array}{cccc}
1 & \varepsilon & 0 & 0 \\
0 & 1 & 0 & 0 \\
0 & 0 & 1 & -\varepsilon \\
0 & 0 & 0 & 1
\end{array}\right), \quad e^{\varepsilon W}=\left(\begin{array}{cccc}
1 & 0 & 0 & 0 \\
0 & \cos \varepsilon & \sin \varepsilon & 0 \\
0 & -\sin \varepsilon \cos \varepsilon & 0 \\
0 & 0 & 0 & 1
\end{array}\right)
$$

Consequently the entries $\left\{a_{i j}\right\}$ of the matrix $M=e^{a(\varepsilon) Z} e^{b(\varepsilon) W} e^{c(\varepsilon) Z} e^{d(\varepsilon) W} e^{f(\varepsilon) Z}$ are found to be

$$
\begin{aligned}
& a_{11}=a_{44}=1, a_{12}=f+c \cos d+a \cos (b+d), a_{13}=c \sin d+a \sin (b+d), \\
& a_{14}=-f c \sin d-a f \sin (b+d)-a c \sin b, a_{22}=a_{33}=\cos (b+d), \\
& a_{23}=-a_{32}=\sin (b+d), a_{24}=-f \sin (b+d)-c \sin b, \\
& a_{34}=-f \cos (b+d)-c \cos b-b, a_{21}=a_{31}=a_{41}=a_{42}=a_{43}=0 .
\end{aligned}
$$

Consequently, the relation $M=e^{\varepsilon Y}$ yields the system of equations

$$
b+d=0, \quad f c \sin d+a c \sin b=0, \quad c \sin d=-\varepsilon, f+c \cos d+a=0 .
$$

whose solution is given by (3.37). 


\subsubsection{Solution using the DE method}

The following identities hold for all $\varepsilon$.

$$
\begin{aligned}
& e^{\varepsilon W} Y \equiv(\cos \varepsilon Y-\sin \varepsilon Z) e^{\varepsilon W}, e^{\varepsilon W} Z \equiv(\cos \varepsilon Z+\sin \varepsilon Y) e^{\varepsilon W} \\
& e^{\varepsilon Z} Y \equiv(Y-\varepsilon X) e^{\varepsilon Z}, e^{\varepsilon Z} W \equiv\left(W-\varepsilon Y+\frac{1}{2} \varepsilon^{2} X\right) e^{\varepsilon Z}
\end{aligned}
$$

After differentiating with respect to $\varepsilon$ the equation $e^{a(\varepsilon) Z} e^{b(\varepsilon) W} e^{c(\varepsilon) Z} e^{d(\varepsilon) W} e^{f(\varepsilon) Z}=e^{\varepsilon Y}$ and repeatedly using the identities (3.39), one obtains the ODE system

$$
\begin{aligned}
& b^{\prime}+d^{\prime}=0, a^{\prime}+\cos b c^{\prime}+c \sin b d^{\prime}+\cos (b+d) f^{\prime}=0, \\
& -a b^{\prime}+\sin b c^{\prime}-(a+c \cos b) d^{\prime}+\sin (b+d) f^{\prime}=1, \\
& \frac{1}{2} a^{2} b^{\prime}-a \sin b c^{\prime}+\left(a c \cos b+\frac{1}{2}\left(a^{2}+c^{2}\right)\right) d^{\prime}-(c \sin d+a \sin (b+d)) f^{\prime}=0 .
\end{aligned}
$$

The solution to the ODE system (3.40) is given by (3.37).

\subsection{Lie algebra $S_{4,9}$}

\subsubsection{Solution using the operator method}

From [10], an operator representation for $S_{4,9}$ is given by

$$
W=\frac{1}{2}\left(y^{2}-z^{2}-4 r x\right) \frac{\partial}{\partial x}-(r y+z) \frac{\partial}{\partial y}+(y-r z) \frac{\partial}{\partial z}, \quad X=\frac{\partial}{\partial x}, \quad Y=\frac{\partial}{\partial y}, \quad Z=y \frac{\partial}{\partial x}+\frac{\partial}{\partial z}
$$

One can show that for all $\varepsilon$ the following relations hold.

$$
\begin{aligned}
e^{\varepsilon Y}(x, y, z)= & (x, y+\varepsilon, z), e^{\varepsilon Z}(x, y, z)=(x+\varepsilon y, y, z+\varepsilon) \\
e^{\varepsilon W}(x, y, z)= & \left(\frac{1}{4}\left(\sin (2 \varepsilon)\left(y^{2}-z^{2}\right)+2 \cos (2 \varepsilon) z y\right)+x-\frac{1}{2} z y\right) e^{-2 \varepsilon r}, e^{-\varepsilon r}(\cos \varepsilon y \\
& \left.-\sin \varepsilon z), e^{-\varepsilon r}(\cos \varepsilon z+\sin \varepsilon y)\right)
\end{aligned}
$$

These relations allow one to show that the equation $e^{a(\varepsilon) Z} e^{b(\varepsilon) W} e^{c(\varepsilon) Z} e^{d(\varepsilon) W} e^{f(\varepsilon) Z}(x, y, z)=e^{\varepsilon Y}(x, y, z)$ leads to the equations

$$
f+c=0, d \sin f+\varepsilon e^{f r}=0, \quad b+g+d \cos f e^{-f r}=0, \quad 2 g \sin f-\varepsilon \cos f=0
$$

The solution to the system of equations (3.41) is given by

$$
a(\varepsilon)=f(\varepsilon)=-\frac{\varepsilon}{2 \tan b(\varepsilon)}, \quad c(\varepsilon)=\frac{\varepsilon e^{-r b}}{\sin b(\varepsilon)}, d(\varepsilon)=-b(\varepsilon),
$$

where $b(\varepsilon)$ is any continuous function chosen so that $a(\varepsilon), f(\varepsilon)$ and $c(\varepsilon)$ are continuous functions, and satisfying $b(\varepsilon) \neq 0$ for any $\varepsilon \neq 0$ with $a(0)=b(0)=c(0)=d(0)=f(0)=0$. 


\subsubsection{Solution using the matrix representation method}

A matrix representation for $S_{4,9}$ is given by

$$
X=\left(\begin{array}{cccc}
0 & 0 & 0 & -2 r \\
0 & 0 & 0 & 0 \\
0 & 0 & 0 & 0 \\
0 & 0 & 0 & 0
\end{array}\right), \quad Y=\left(\begin{array}{cccc}
0 & 0 & 1 & 0 \\
0 & 0 & 0 & -r \\
0 & 0 & 0 & 1 \\
0 & 0 & 0 & 0
\end{array}\right), \quad Z=\left(\begin{array}{cccc}
0 & -1 & 0 & 0 \\
0 & 0 & 0 & -1 \\
0 & 0 & 0 & -r \\
0 & 0 & 0 & 0
\end{array}\right), \quad W=\left(\begin{array}{cccc}
2 r & 0 & 0 & 0 \\
0 & r & 1 & 0 \\
0 & -1 & r & 0 \\
0 & 0 & 0 & 0
\end{array}\right)
$$

The corresponding matrix representation of the associated Lie group is given by

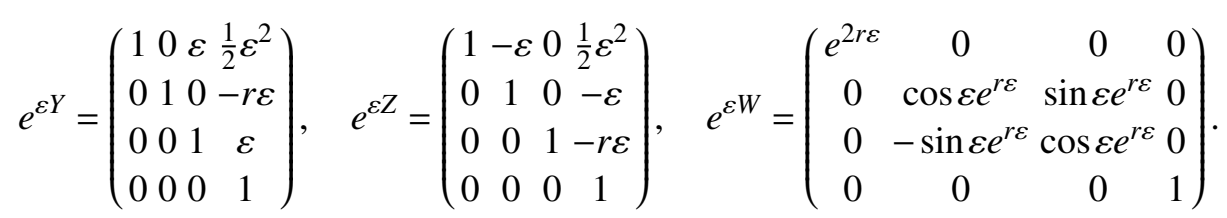

Consequently the entries $\left\{a_{i j}\right\}$ of the matrix $M=e^{a(\varepsilon) Z} e^{b(\varepsilon) W} e^{c(\varepsilon) Z} e^{d(\varepsilon) W} e^{f(\varepsilon) Z}$ are given by

$$
\begin{aligned}
& a_{11}=e^{2 r(b+d)}, \\
& a_{12}=-f e^{2 r(b+d)}-c \cos d e^{r(2 b+d)}-a \cos (b+d) e^{r(b+d)}, \\
& a_{13}=-c \sin d e^{r(2 b+d)}-a \sin (b+d) e^{r(b+d)}, \\
& a_{14}=\frac{1}{2} f^{2} e^{2 r(b+d)}+c f \cos d e^{r(2 b+d)}+r c f \sin d e^{r(2 b+d)}+a f \cos (b+d) e^{r(b+d)}+ \\
& r a f \sin (b+d) e^{r(b+d)}+\frac{1}{2} c^{2} e^{2 r b}+a c \cos b e^{r b}+r a c \sin b e^{r b}+\frac{1}{2} a^{2}, \\
& a_{22}=a_{33}=\cos (b+d) e^{r(b+d)}, a_{23}=-a_{32}=\sin (b+d) e^{r(b+d)}, \\
& a_{24}=-f \cos (b+d) e^{r(b+d)}-r f \sin (b+d) e^{r(b+d)}-c \cos b e^{r b}-r c \sin b e^{r b}-a, \\
& a_{34}=f \sin (b+d) e^{r(b+d)}-r f \cos (b+d) e^{r(b+d)}+c \sin b e^{r b}-r c \cos b e^{r b}-r b, \\
& a_{44}=1, a_{21}=a_{31}=a_{41}=a_{42}=a_{43}=0 .
\end{aligned}
$$

The relation $M=e^{\varepsilon Y}$ yields, after simplification, the system of equations

$$
\begin{aligned}
& b+d=0, \quad c \sin b e^{r b}=\varepsilon, f+c \cos d e^{r b}+a=0, \\
& \frac{1}{2} f^{2}+(a+f) c \cos b e^{r b}+r(a-f) c \sin b e^{r b}+a f+\frac{1}{2} c^{2} e^{2 r b}+\frac{1}{2} a^{2}=\frac{1}{2} \varepsilon^{2} .
\end{aligned}
$$

The solution to the system of equations (3.43) is given by (3.41).

\subsubsection{Solution using the DE method}

The following identities hold for all $\varepsilon$.

$$
\begin{aligned}
e^{\varepsilon W} Y & \equiv\left(\cos \varepsilon e^{r \varepsilon} Y-\sin \varepsilon e^{r \varepsilon} Z\right) e^{\varepsilon W}, e^{\varepsilon W} Z \equiv\left(\cos \varepsilon e^{r \varepsilon} Z+\sin \varepsilon e^{r \varepsilon} Y\right) e^{\varepsilon W}, \\
e^{\varepsilon W} X & \equiv e^{2 r \varepsilon} X e^{\varepsilon W}, e^{\varepsilon Z} Y \equiv(Y-\varepsilon X) e^{\varepsilon Z}, e^{\varepsilon Z} W \equiv\left(W-r \varepsilon Z-\varepsilon Y+\frac{1}{2} \varepsilon^{2} X\right) e^{\varepsilon Z}, \\
e^{\varepsilon Z} X & \equiv X e^{\varepsilon Z} .
\end{aligned}
$$


After differentiating with respect to $\varepsilon$ the equation $e^{a(\varepsilon) Z} e^{b(\varepsilon) W} e^{c(\varepsilon) Z} e^{d(\varepsilon) W} e^{f(\varepsilon) Z}=e^{\varepsilon Y}$ and using the identities (3.44) one obtains the ODE system

$$
\begin{aligned}
& b^{\prime}+d^{\prime}=0, a^{\prime}+\cos b e^{r b} c^{\prime}+\left(-r c \cos b e^{r b}+c \sin b e^{r b}\right) d^{\prime}+e^{r(b+d)} f^{\prime}=0, \\
& -a b^{\prime}+\sin b e^{r b} c^{\prime}+\left(-r c \sin b e^{r b}-c \cos b e^{r b}\right) d^{\prime}=1, \\
& -a \sin b e^{r b} c^{\prime}+\left(r a c \sin b e^{r b}+a c \cos b e^{r b}+\frac{1}{2} c^{2} e^{2 r b}\right) d^{\prime}-c \sin d e^{r b} f^{\prime}=0 .
\end{aligned}
$$

The solution to the ODE system (3.44) is given by (3.41).

\subsection{Lie algebra $S_{4,10}$}

\subsubsection{Solution using the operator method}

From [10], an operator representation for $S_{4,10}$ is given by

$$
W=-2 x \frac{\partial}{\partial x}-y \frac{\partial}{\partial y}+\frac{\partial}{\partial z}, X=\frac{\partial}{\partial x}, Y=\frac{\partial}{\partial y}, Z=y \frac{\partial}{\partial x}+z \frac{\partial}{\partial y} .
$$

Consequently, the equation $e^{a(\varepsilon) W} e^{b(\varepsilon) Z} e^{c(\varepsilon) W} e^{d(\varepsilon) Z} e^{f(\varepsilon) W} e^{g(\varepsilon) W}(x, y, z)=e^{\varepsilon Y}(x, y, z)$ leads to the simplified system of equations

$$
\begin{aligned}
& a+c+f=0, \quad g+d e^{a+c}+b e^{a}=0, \quad c d e^{-f}+c g+f g=\varepsilon, \\
& d^{2} c e^{-2 f}+2 g d c e^{-f}-a g^{2}=0,
\end{aligned}
$$

whose solutions are given by

$$
\begin{aligned}
& f(\varepsilon)=-(a(\varepsilon)+c(\varepsilon)), \quad b(\varepsilon)=\varepsilon e^{-a}\left(\frac{c(\varepsilon) \pm \sqrt{c(\varepsilon)^{2}+a(\varepsilon) c(\varepsilon)}}{a(\varepsilon) c(\varepsilon)}\right) \\
& g(\varepsilon)=-\frac{\varepsilon+b(\varepsilon) c(\varepsilon) e^{a(\varepsilon)}}{a(\varepsilon)+c(\varepsilon)}, d(\varepsilon)=-g(\varepsilon) e^{-(a(\varepsilon)+c(\varepsilon))}-b(\varepsilon) e^{-c(\varepsilon)}
\end{aligned}
$$

where $a(\varepsilon)$ and $c(\varepsilon)$ are any continuous functions chosen so that $b(\varepsilon), d(\varepsilon)$ and $g(\varepsilon)$ are continuous functions, and satisfying $a(\varepsilon) c(\varepsilon) \neq 0, a(\varepsilon)+c(\varepsilon) \neq 0$, and $c(\varepsilon)^{2}+a(\varepsilon) c(\varepsilon) \geq 0$ for any $\varepsilon \neq 0$ with $a(0)=b(0)=c(0)=d(0)=f(0)=g(0)=0$.

In the limiting case when $a(\varepsilon)=0, f(\varepsilon)=-c(\varepsilon), d(\varepsilon)=\frac{\varepsilon e^{-c(\varepsilon)}}{c(\varepsilon)}, b(\varepsilon)=g(\varepsilon)=-\frac{\varepsilon}{2 c(\varepsilon)}$, with $c(\varepsilon)$ any continuous function chosen so that $b(\varepsilon), g(\varepsilon)$ and $d(\varepsilon)$ are continuous functions, and $c(\varepsilon) \neq 0$ when $\varepsilon \neq 0$.

\subsubsection{Solution using the matrix representation method}

A matrix representation of $S_{4,10}$ is given by

$$
X=\left(\begin{array}{cccc}
0 & 0 & 0 & -2 \\
0 & 0 & 0 & 0 \\
0 & 0 & 0 & 0 \\
0 & 0 & 0 & 0
\end{array}\right), \quad Y=\left(\begin{array}{cccc}
0 & 0 & 1 & 0 \\
0 & 0 & 0 & -1 \\
0 & 0 & 0 & 0 \\
0 & 0 & 0 & 0
\end{array}\right), \quad Z=\left(\begin{array}{cccc}
0 & -1 & 0 & 0 \\
0 & 0 & 0 & -1 \\
0 & 0 & 0 & -1 \\
0 & 0 & 0 & 0
\end{array}\right), \quad W=\left(\begin{array}{cccc}
2 & 0 & 0 & 0 \\
0 & 1 & 1 & 0 \\
0 & 0 & 1 & 0 \\
0 & 0 & 0 & 0
\end{array}\right)
$$


One can show that

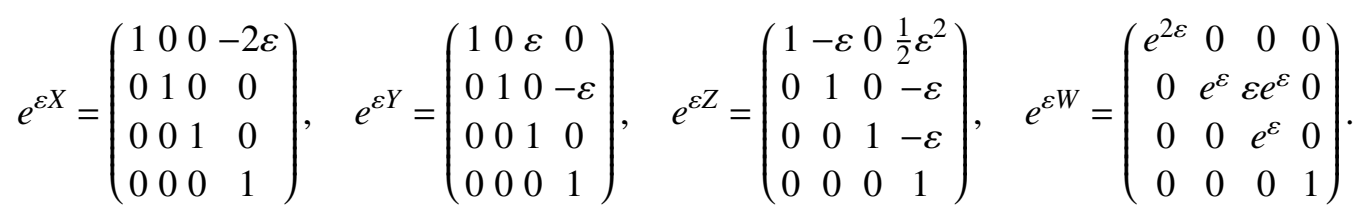

Then the matrix $M=e^{a(\epsilon) W} e^{b(\epsilon) Z} e^{c(\epsilon) W} e^{d(\epsilon) Z} e^{f(\varepsilon) W} e^{g(\varepsilon) Z}$ has entries $\left\{a_{i j}\right\}$ given by

$$
\begin{aligned}
& a_{11}=e^{2(a+c+f)}, a_{12}=-\left(g e^{c+f}+d e^{c}+b\right) e^{2 a+c+f}, a_{13}=-\left(f d e^{c}+f b+b c\right) e^{2 a+c+f}, \\
& a_{14}=\left(\frac{1}{2} g^{2} e^{c+f}+g(1+f) d e^{c}+g b(1+f)+b c g\right) e^{2 a+c+f}+\left(\frac{1}{2} d^{2} e^{2 c}+b d(1+c) e^{c}+\frac{1}{2} b^{2}\right) e^{2 a}, \\
& a_{22}=a_{33}=e^{a+c+f}, a_{23}=(a+c+f) e^{a+c+f}, \\
& a_{24}=-g(1+a+c+f) e^{a+c+f}-\left(d(1+a+c) e^{c}+b(1+a)\right) e^{a}, \\
& a_{34}=-\left(g e^{a+c+f}+d e^{a+c}+b e^{a}\right), a_{44}=1, a_{i j}=0 \text { for } j<i .
\end{aligned}
$$

Consequently, after simplification, the relation $M=e^{\varepsilon Y}$ leads to the system of equations (3.46) whose solutions are given by (3.47).

\subsubsection{Solution using the DE method}

One can show that the following identities hold for all $\varepsilon$.

$$
\begin{aligned}
e^{\varepsilon W} X & \equiv\left(e^{2 \varepsilon} X\right) e^{\varepsilon W}, e^{\varepsilon W} Y \equiv\left(e^{\varepsilon} Y\right) e^{\varepsilon W}, e^{\varepsilon W} Z \equiv\left(e^{\varepsilon} Z+\varepsilon e^{\varepsilon} Y\right) e^{\varepsilon W}, e^{\varepsilon Z} X \equiv X e^{\varepsilon Z}, \\
e^{\varepsilon Z} Y & \equiv(Y-\varepsilon X) e^{\varepsilon Z}, e^{\varepsilon Z} W \equiv\left(W-\varepsilon Z-\varepsilon Y+\frac{1}{2} \varepsilon^{2} X\right) e^{\varepsilon Z} .
\end{aligned}
$$

After differentiating with respect to $\varepsilon$ the equation $e^{a(\varepsilon) W} e^{b(\varepsilon) Z} e^{c(\varepsilon) W} e^{d(\varepsilon) Z} e^{f(\varepsilon) W} e^{g(\varepsilon) Z}=e^{\varepsilon Y}$ and using the identities in (3.48), one obtains the ODE system

$$
\begin{aligned}
& a^{\prime}+c^{\prime}+f^{\prime}=0, \\
& e^{a}\left(a b^{\prime}-b(1+a) c^{\prime}+(a+c) e^{c} d^{\prime}-\left(a b+a d e^{c}+b+d(1+c) e^{c}\right) f^{\prime}\right)=1, \\
& e^{a} b^{\prime}-b e^{a} c^{\prime}+e^{a+c} d^{\prime}-\left(b+e^{c} d\right) e^{a} f^{\prime}+g^{\prime}=0, \\
& \frac{1}{2} b^{2} e^{2 a} c^{\prime}-b c e^{2 a+c} d^{\prime}+\left(\frac{1}{2}\left(b^{2}+d^{2} e^{2 c}\right)+b d(1+c) e^{c}\right) e^{2 a} f^{\prime}-\left((b f+b c) e^{f+c+2 a}+\right. \\
& \left.d f e^{f+2 c+2 a}\right) g^{\prime}=0 .
\end{aligned}
$$

The solution to ODE system (3.48) is given by (3.47).

\section{Discussion and conclusions}

In this paper, for all three- and four-dimensional Lie algebras satisfying (2.1) with $C_{12}^{3} \neq 0$ and (2.5), respectively, we have shown explicitly how one can obtain elements of the associated Lie groups as compositions of products of other elements from the commutator properties of their Lie algebras. Three methods have been presented to accomplish this: an operator method, a matrix representation method, and a DE method. It turns out that in all cases solutions contain an arbitrary function of a parameter $\varepsilon$. In the parallel parking problem, the parameter $\varepsilon$ is a translation in $x$ arising from translations in $y$ and rotations in the $x y$-plane and the arbitrary continuous function can be the angle of rotation or the initial translation. Interestingly, in all cases solutions can be expressed in terms 
of elementary functions involving an arbitrary continuous function. In practical applications, other constraints could be satisfied by appropriately restricting associated arbitrary functions.

There is an "initial condition" that constrains the arbitrary function. In particular as $\varepsilon \rightarrow 0$, if the arbitrary function is $O\left(\varepsilon^{p}\right)$ then it is easy to check that $0<p<1$ and that all other functions in the compositions are either $O\left(\varepsilon^{p}\right)$ or $O\left(\varepsilon^{1-p}\right)$.

As noted earlier in the paper, one can also state the problems in (2.2) and (2.6) with the roles of $B_{1}$ and $B_{2}$ interchanged. It was found that doing so, when the number of terms to the left of equations (2.2) and (2.6) is even, does not significantly change the solutions. For example, in the parallel parking problem, considering the equation $e^{a(\varepsilon) R} e^{b(\varepsilon) Y} e^{c(\varepsilon) R} e^{d(\varepsilon) Y}=e^{\varepsilon X}$ leads to the solution

$$
a(\varepsilon)=-c(\varepsilon), \quad b(\varepsilon)=-\frac{\varepsilon}{\sin c(\varepsilon)}, d(\varepsilon)=\varepsilon \cot c(\varepsilon),
$$

where, for any $\varepsilon \neq 0, c(\varepsilon)$ is any continuous function chosen so that $b(\varepsilon)$ and $d(\varepsilon)$ are continuous, and satisfying $c(\varepsilon) \neq k \pi$ for every $k \in \mathbb{Z}$ with $a(0)=b(0)=c(0)=d(0)=0$. On the other hand, considering the alternative equation $e^{a(\varepsilon) Y} e^{b(\varepsilon) R} e^{c(\varepsilon) Y} e^{d(\varepsilon) R}=e^{\varepsilon X}$ leads to the solution

$$
d(\varepsilon)=-b(\varepsilon), c(\varepsilon)=\frac{\varepsilon}{\sin b(\varepsilon)}, a(\varepsilon)=-\varepsilon \cot b(\varepsilon),
$$

where $b(\varepsilon)$ is any continuous function chosen so that $a(\varepsilon)$ and $c(\varepsilon)$ are continuous, and satisfying both $b(\varepsilon) \neq k \pi$ for every $k \in \mathbb{Z}$ and every $\varepsilon \neq 0$ and $a(0)=b(0)=c(0)=d(0)=0$. As one can observe, the two solutions are clearly isomorphic. However, if the number of terms to the left of (2.2) and (2.6) is odd, then interchanging the roles of $B_{1}$ and $B_{2}$ may lead to a problem with no solutions. For instance, considering the $S_{4,7}$ problem with the alternative order of $Z$ and $W$ leads to no solutions.

Each of the three methods, used to solve equations (2.2) and (2.6), have different strengths and challenges. When a useful operator representation exists, the operator method offers a computationally very simple and complete approach to solving (2.2) and (2.6). However, an appropriate operator representation of a Lie algebra is only known for three- and four-dimensional Lie algebras [10]. But one would expect an operator representation to exist for Lie algebras that arise in practical problems.

The matrix representation method requires a matrix representation of a Lie algebra. Such a representation may not always be readily available. In the case of $S_{4,7}$, for example, the matrix representation found using the software [7] was not faithful and thus could not be used. Instead, our correction of the adjoint matrix representation found in [4] was used. Another issue with the matrix representation method is that the software GAP [7] cannot handle Lie algebras with algebraic values or non-integers in their structure constants. Accordingly, we had to make adjustments for the matrix representations for the Lie algebras $S_{3,1}, S_{3,3}$, and, $S_{4,9}$. Moreover, without carrying out all calculations, the number of independent equations one obtains from the matrix representation method and whether a solution exists cannot be determined a priori. The main strength of the matrix representation method is that in all cases it resulted in algebraic systems of equations that we were able to solve. Most importantly, the matrix representation method is complete since it leads to necessary and sufficient conditions for solutions.

Unlike the matrix and operator representation methods, the differential equation method (DE method) requires no Lie algebra representation. Moreover, it can handle all forms of structure constants. Furthermore, in the DE method, unlike the other two methods, one can see that the solution should depend on an arbitrary function before calculations are performed since the resulting system of ODEs has more unknowns than the number of ODEs in the system. However, the resulting first order system of nonlinear ODEs often presents a more significant challenge to solve than the 
system of equations obtained through the other two methods. For instance, we were unable to solve directly the ODE system associated with the Euler angles problem but obtained its solution using the operator and matrix representation methods. The most crucial issue with the DE method remains that it only yields a necessary condition. But for all cases considered, it turns out that the obtained solutions satisfied both necessary and sufficient conditions. Related to this, it is an open problem to prove the existence and uniqueness theorem for the nonlinear systems of first order ODEs that result from the DE method for any relevant $n$-dimensional Lie algebra without use of solutions arising from matrix or operator representations.

One should note that it is possible to extend the solutions presented in this paper by not requiring the initial conditions (2.19) or (2.20) to be satisfied. As examples, the parallel parking problem also has the solution

$$
d(\varepsilon)=\varepsilon, c(\varepsilon)=-a(\varepsilon)=\frac{\pi}{4}, \quad b(\varepsilon)=\sqrt{2} \varepsilon ;
$$

the Euler angles problem also has the solution (3.11).

It is of interest to note that the operator and matrix representation methods are algebraic ways of solving nonlinear ODE systems arising from the DE method!

\section{Acknowledgments}

The authors acknowledge financial support from the Natural Sciences and Engineering Research Council of Canada. We are grateful for the remarks of the referees and to Zinovy Reichstein for helpful discussions. Most importantly, we thank one of the referees for making us aware of Reference [10].

\section{References}

[1] I.D. Ado, The representation of Lie algebras by matrices. Amer. Math. Soc. Translations, 2,21 pp. (1949).

[2] G. Bluman, Construction of Solutions to Partial Differential Equations by the Use of Transformation Groups, Ph.D. Thesis, Caltech (1967).

[3] R.W. Brockett, Lie algebras and Lie groups in control theory, Geometric Methods in System Theory: Proceedings of the NATO Advanced Study Institute, (Dordrecht: D. Reidel Pub. Co., D.Q. Mayne and R.W. Brockett eds., 1973), pp. 43-82 (1973).

[4] R. Ghanam and G. Thompson, Minimal matrix representation of four-dimensional Lie algebras. Bull. Malays. Math. Sci. Soc. 36, 343-349 (2013).

[5] R. Ghanam and G. Thompson, Minimal matrix representation of four-dimensional Lie algebras. Extracta Math. 30, 95-133 (2015).

[6] A. González-López, N. Kamran, and P. Olver, Lie algebras of differential operators in two complex variables. Amer. J. Math. 114, 1163-1185 (1992).

[7] W.A. de Graaf, Constructing Faithful Matrix Representations of Lie Algebras, Proceedings of the 1997 International Symposium on Symbolic and Algebraic Computation, pp. 54-59 (1997).

[8] E. Kirillova and K. Spindler, Optimal control of a vehicle during parking. IFAC Proceedings Volumes, 37 (13), 925-930 (2004).

[9] E. Nelson, Tensor Analysis, (Princeton University Press, Princeton, NJ, 1967).

[10] R.O. Popovych, V.M. Boyko, M.O. Nesterenko, and M.W. Lutfullin, Realizations of real lowdimensional Lie algebras. J. Phys. A 36, 7337-7360 (2003).

[11] L. Snobl and P. Winternitz, Classification and Identification of Lie Algebras, (American Mathematical Society, Providence, RI, 2014). 